\title{
Tuning Nuclear Quadrupole Resonance: A Novel Approach for the Design of Frequency-Selective MRI Contrast Agents
}

\author{
Christian Gösweiner, ${ }^{1, *}$ Perttu Lantto, ${ }^{2}$ Roland Fischer, ${ }^{3}$ Carina Sampl, ${ }^{3}$ Evrim Umut, ${ }^{4}$ Per-Olof Westlund, ${ }^{5}$ \\ Danuta Kruk, ${ }^{4}$ Markus Bödenler, ${ }^{1}$ Stefan Spirk ${ }^{6,7}$ Andreas Petrovič, ${ }^{1}$ and Hermann Scharfetter ${ }^{1}$ \\ ${ }^{1}$ Institute of Medical Engineering, Graz University of Technology, 8010 Graz, Austria \\ ${ }^{2}$ NMR Research Unit, University of Oulu, P.O. Box 3000, 90014 Oulu, Finland \\ ${ }^{3}$ Institute of Inorganic Chemistry, Graz University of Technology, 8010 Graz, Austria \\ ${ }^{4}$ Faculty of Mathematics and Computer Science, University of Warmia and Mazury in Olsztyn, \\ 10-719 Olsztyn, Poland \\ ${ }^{5}$ Departement of Chemistry, Umeå University, 90187 Umeå, Sweden \\ ${ }^{6}$ Institute for Chemistry and Technology of Materials, Graz University of Technology, 8010 Graz, Austria \\ ${ }^{7}$ Institute of Paper, Pulp and Fibre Technology, Graz University of Technology, 8010 Graz, Austria
}

(Received 16 January 2018; revised manuscript received 6 April 2018; published 25 June 2018)

\begin{abstract}
The interaction between water protons and suitable quadrupolar nuclei (QN) can lead to quadrupole relaxation enhancement (QRE) of proton spins, provided the resonance condition between both spin transitions is fulfilled. This effect could be utilized as a frequency selective mechanism in novel, responsive $T_{1}$ shortening contrast agents (CAs) for magnetic resonance imaging (MRI). In particular, the proposed contrast mechanism depends on the applied external flux density — a property that can be exploited by special field-cycling MRI scanners. For the design of efficient CA molecules, exhibiting narrow and pronounced peaks in the proton $T_{1}$ relaxation dispersion, the nuclear quadrupole resonance (NQR) properties, as well as the spin dynamics of the system $\mathrm{QN}-{ }^{1} \mathrm{H}$, have to be well understood and characterized for the compounds in question. In particular, the energy-level structure of the $\mathrm{QN}$ is a central determinant for the static flux densities at which the contrast enhancement appears. The energy levels depend both on the QN and the electronic environment, i.e., the chemical bonding structure in the CA molecule. In this work, the NQR properties of a family of promising organometallic compounds containing ${ }^{209} \mathrm{Bi}$ as $\mathrm{QN}$ have been characterized. Important factors like temperature, chemical structure, and chemical environment have been considered by NQR spectroscopy and $a b$ initio quantum chemistry calculations. The investigated Bi-aryl compounds turned out to fulfill several crucial requirements: NQR transition frequency range applicable to clinical 1.5- and $3 \mathrm{~T}$ MRI systems, low temperature dependency, low toxicity, and tunability in frequency by chemical modification.
\end{abstract}

DOI: 10.1103/PhysRevX.8.021076

\section{INTRODUCTION}

Magnetic resonance imaging (MRI) is one of the most powerful diagnostic imaging tools in modern medicine. The technique features high spatial resolution combined with high penetration depth and superb soft tissue contrast without the use of ionizing radiation. Tissue contrast in MRI is essentially based on the distribution of water protons within the body (proton density), as well as their

\footnotetext{
* Corresponding author. christian.goesweiner@tugraz.at

Published by the American Physical Society under the terms of the Creative Commons Attribution 4.0 International license. Further distribution of this work must maintain attribution to the author(s) and the published article's title, journal citation, and DOI.
}

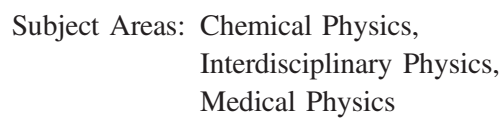

spin-lattice and spin-spin relaxation times $\left(T_{1}, T_{2}\right)$. To further increase contrast and, consequently, sensitivity, signal enhancement strategies have been developed involving the use of relaxation enhancers, so-called contrast agents (CAs). The most widely used CAs are paramagnetic chelates based on gadolinium $(\mathrm{Gd})$ that shorten the $T_{1}$ of free water protons [1]. In contrast, nanoparticles composed of paramagnetic iron oxides predominantly shorten $T_{2}$ [2]. These CAs are administered to patients, leading to an improved soft tissue contrast in either $T_{1}$ or $T_{2}$ weighted sequences. As a consequence, MRI is nowadays employed for both morphological and functional imaging, such as dynamic contrast enhancement (DCE) in cancer diagnostics [3]. MRI is increasingly used also in the context of cellular and molecular imaging [4], the aim of which is to provide spatially and temporally resolved maps of biomarkers, which contain information on pathophysiological 
processes correlated with cancer and other diseases [5]. This is usually achieved by administration of CAs that respond to their chemical environment. Considerable effort has been directed into the development of MRI CAs exhibiting a significant change in relaxivity upon activation in response to physiological alterations such as temperature, metal ions, redox state, enzyme activity, or $\mathrm{pH}[6,7]$.

One representative of such responsive CAs is based on chemical exchange saturation transfer (CEST) [8-10], which has gained more and more interest in recent years. CEST contrast can be switched on and off using a selective radio frequency $(\mathrm{RF})$ saturation pulse. However, CEST suffers from low sensitivity, SAR restrictions, and saturation spillover effects between bulk protons and protons of the CEST agent, which are due to insufficient frequency separation. Also, new nitroxide-based macromolecules have been reported as $T_{2}$ shortening agents [11], which provide a system free of toxic heavy metals. As they contain radicals, their in vivo lifetime may become a limiting factor, and they are not inherently switchable.

In this paper, we explore a novel alternative approach to the established CAs making use of quadrupole relaxation enhancement (QRE) [12-14] induced by the interaction of protons with quadrupolar nuclei (i.e., nuclei with spin quantum number $I>1 / 2$ ) [15-17]. The ability of QRE to enhance MRI contrasts has been proven for the interaction between endogenous ${ }^{1} \mathrm{H}$ and ${ }^{14} \mathrm{~N}$ in the amide groups of muscle proteins at very low flux densities [18]. However, it is completely unexplored for exogenous CAs at clinical fields, such as 1.5 or $3 \mathrm{~T}$.

For QRE, the dipole-dipole (D-D) interaction between a proton spin and the nuclear spin of a quadrupolar nucleus (QN) is associated with a transfer of magnetization from the proton to the QN and, thus, accelerates the proton spin relaxation. Designing a CA based on QRE, however, is a complex task, as several conditions concerning, e.g., the ${ }^{1} \mathrm{H}-\mathrm{QN}$ distance and the timescale of the motional dynamics must be met for the phenomenon to take place. In particular, the process becomes effective when the proton spin transition frequency (i.e., the Larmor frequency) matches to one of the spin transition frequencies of the $\mathrm{QN}$, which depend on its quadrupole coupling constant $Q_{c c}$ and the Zeeman splitting.

QRE-based CAs are selective to $B_{0}$ due to the field dependence of this resonance condition. Moreover, $Q_{c c}$ is sensitive to the temperature as well as the chemical surrounding of the QN and, subsequently, to subtle changes of the bonding structure, e.g., by chemical reactions. Thus, contrast can be modulated, e.g., either by altering the chemical structure of the $\mathrm{CA}$ or by shifting the main magnetic field $B_{0}$ of a MRI system.

Utilizing the combination of fast field cycling (FFC) relaxometry with MRI (FFC-MRI), it is possible to cycle the nominal $B_{0}$ field during an imaging sequence $[18,19]$. This technique gives access to contrast types arising from the field dependency of the proton relaxation rates $R_{1}\left(B_{0}\right)=1 / T_{1}\left(B_{0}\right)$ and $R_{2}\left(B_{0}\right)=1 / T_{2}\left(B_{0}\right)$, referred to as nuclear magnetic relaxation dispersion (NMRD). CAs exhibiting a strong $R_{1}$ dispersion are especially favorable to be imaged by delta relaxation enhanced magnetic resonance (dreMR) imaging [20,21], as the $R_{1}$ dispersion of healthy tissue is inherently low at clinical fields [22]. QRE is in principle capable of generating narrow peaks instead of just a smooth slope in the proton $T_{1}$-NMRD profile, so it offers the possibility to modulate contrast by cycling the field of the scanner.

The first step of the development of a potential contrast agent is the identification of promising chemical compounds that contain a suitable QN.

The basic selection criteria are

(i) high gyromagnetic ratio $\gamma_{\mathrm{QN}}$ to provide strong D-D coupling and hence strong QRE

(ii) high nuclear spin I, preferably $I=9 / 2$, as the relaxation rate $R_{1}$ scales with $I$ [23] and a higher nuclear spin offers more possible quadrupole transition frequencies that can fulfill the resonance condition

(iii) low toxicity

(iv) high natural abundance

(v) rich and well-known chemistry

(vi) high $Q_{c c}$, so that there exist transition frequencies close to the Larmor frequency at clinical field strengths $(1.5$ or $3 \mathrm{~T}) . Q_{c c}$ depends on both the quadrupolar moment of the QN and the electric field gradient (EFG) produced by the electronic environment of the QN, i.e., the chemical bonding structure of the compound.

Among a number of potential QNs, ${ }^{209} \mathrm{Bi}$ with $I=9 / 2$ appears particularly favorable because it is considered to be comparatively nontoxic, and so $\mathrm{Bi}$ compounds are expected to have a higher biocompatibility [24] than, e.g., Gd-based CAs [25,26]. ${ }^{209} \mathrm{Bi}$ has been suggested [27-29] also for $\mathrm{X}$-ray contrast agents, which makes it an ideal candidate for dual-mode CAs for computer tomography (CT) and MRI, with only a single core element.

The knowledge of the nuclear quadrupole resonance (NQR) parameter $Q_{c c}$ and $\eta$ (the asymmetry parameter of the EFG; see Appendix A) is of central importance for fulfilling the QRE resonance condition as they determine the NQR transition spectrum. The purpose of this paper is, thus, to characterize, in terms of these parameters, a set of Bi-aryl compounds that are promising starting candidates for the development of QRE-based contrast agents. Moreover, it is pivotal to identify and, if possible, quantify all relevant mechanisms that can modify these parameters during the development cycle of the final CA.

In this context, the aim of this paper is twofold:

(1) Experimental characterization of $Q_{c c}$ and $\eta$ of a promising set of quadrupolar compounds in their crystalline state by means of zero-field nuclear quadrupole resonance spectroscopy (NQRS) [30] 
(see Sec. III B). As a side product, the quadrupole spin relaxation times $T_{1, Q}$ and $T_{2, Q}$ are also determined.

(2) Estimation of the influence of structural changes on $Q_{c c}$ and $\eta$, when passing from the crystalline state to single isolated molecules in solution (it is not possible to observe NQR peaks directly in liquids [31] due to temporal averaging of the tumbling molecules). This may serve as a first approximation for the situation in the final CA and is achieved by $a b$ initio quantum chemistry (QC) calculations (see Sec. III C).

The paper is organized as follows: First, the basic theoretical background on QRE in protons (Sec. II) is given. Then, the proposed compounds are introduced, and the results on the two main points introduced above are discussed (Sec. III). In Sec. IV, the main conclusions are summarized and, in Sec. V, a brief outlook will be given. At the end of the paper, the methods used for QC calculation and NQR spectroscopy (Sec. VI) are described in detail. Appendix A contains the mathematical description of the NQR transition frequency spectrum. Details of the data analysis and fitting procedure are given in Appendix B. In Appendix C, results and related error estimations are summarized.

\section{QUADRUPOLE RELAXATION ENHANCEMENT IN PROTONS}

First, it is important to recollect the basics of NQR. The interaction of a high-spin nucleus $(I>1 / 2)$ with the EFG generated by its surrounding charge distribution gives rise to a quantized energy level system that can be studied by NQRS $[30,32,33]$. The emerging NQR transition frequency spectrum $\nu_{Q, k}$ (pure quadrupole transition frequency; see Appendix A) can be fully described by $Q_{c c}$ and $\eta$. The number of transitions depends on the nuclear spin quantum number $I$; in the case of ${ }^{209} \mathrm{Bi}$, four pure quadrupole peaks (no external field $B_{0}$ ) can be observed [see Fig. 1(a)]. The NQR transitions provide a very direct and highly sensitive insight into the electronic environment of the molecule where the resonant QN is located. The position of the transition frequency is sensitive to chemical or structural changes of the molecule as well as to the temperature. If the relevant influences are well understood, the transition frequency of the QN can be tuned to a desired frequency and its response upon slight chemical changes can be predicted. A more detailed mathematical deduction of the NQR transition frequency spectrum and the origin of its parameters $Q_{c c}$ and $\eta$ is given in Appendix A.

As soon as an external field $B_{0}$ is applied, QNs and protons experience a splitting of their energy level systems due to Zeeman coupling [see Fig. 1(b)]. In consequence, the degeneracy in the magnetic quantum number $m_{I}$ is canceled, which leads to an observable peak at the Larmor frequency $\nu_{L}$ for protons as well as to a splitting of the pure NQR transition frequencies. The Zeeman contribution to the two

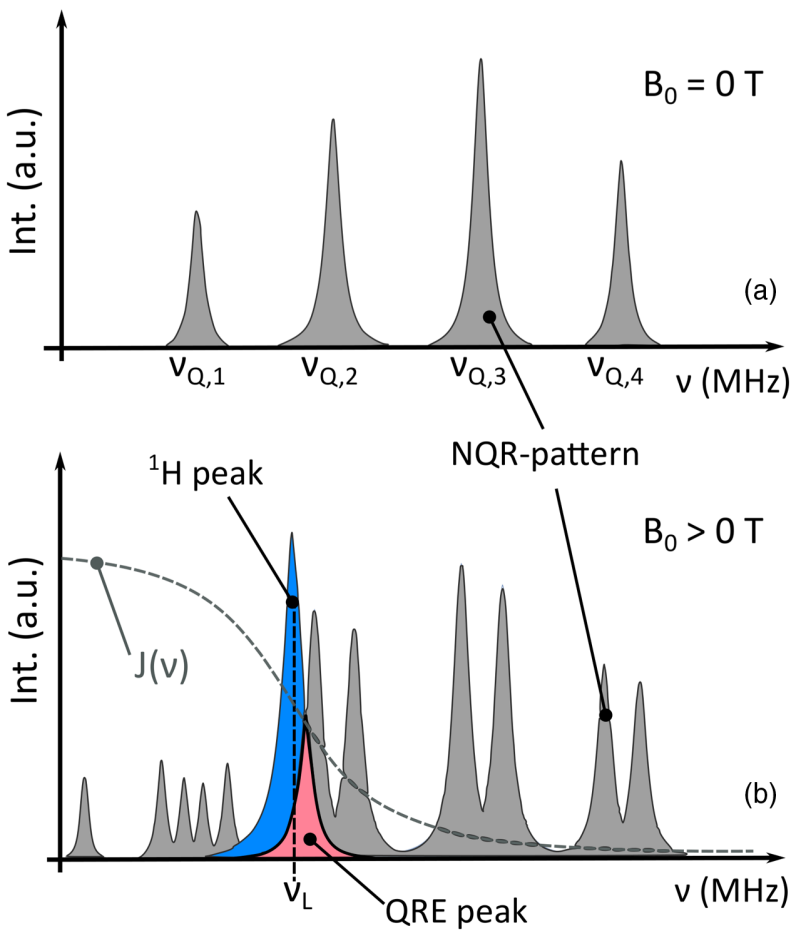

FIG. 1. Simple scheme of transition frequency crossing to produce QRE in protons. For illustration and simplicity, the line shapes are assumed to be Lorentzian [see Appendix B, Eq. (B3)] with equal width for each transition. Intensities in a.u. correspond to Boltzmann weighted transition probabilities [Appendix A, Eq. (A8)]. In panel (a), the pure NQR pattern of a single crystal is shown (grey pattern). In panel (b), $B_{0}>0 \mathrm{~T}$ leads to an observable ${ }^{1} \mathrm{H}$ resonance (blue peak) at the Larmor frequency $\nu_{L}$, as well as to a splitting of the NQR pattern [Appendix A, Eq. (A7)]. The four-line splitting of the lowest transition is a consequence of nonzero off-diagonal elements of the Hamiltonian [30]. A transition frequency match at a particular field strength $B_{0}$-indicated by the red highlighted overlap area-and the D-D fluctuations $J(\nu)$ at the corresponding frequency, are the main conditions for a $\mathrm{QRE}$ of the protons.

spin ensembles depends on $\gamma^{1}{ }_{\mathrm{H}}$ and $\gamma_{\mathrm{QN}}$, respectively. For the QN, the angle $\Omega$ between its EFG and $B_{0}$ has to be considered as well (for the definition, see Appendix A). So, at particular flux densities, it is possible that spin transitions of both ensembles overlap, as indicated by the red area in Fig. 1(b). This is the primary condition for QRE.

There is, however, still another important prerequisite for QRE to occur. As illustrated in Fig. 2(a), fluctuating D-D interactions must act as a coupling mechanism, because QRE is based on a magnetization transfer from a ${ }^{1} \mathrm{H}$ spin ensemble to a quadrupolar spin ensemble by cross relaxation [23]. Therefore, it is required that (1) the ${ }^{1} \mathrm{H}-\mathrm{QN}$ distance $r_{\mathrm{H}-\mathrm{Bi}}$ be small enough and that (2) the spectrum of the D-D fluctuations, which can be described by the spectral density function $J(\nu)$, contain strong components at those frequencies where the resonance condition is met [compare Fig. 1(b)]. The latter condition depends on the 


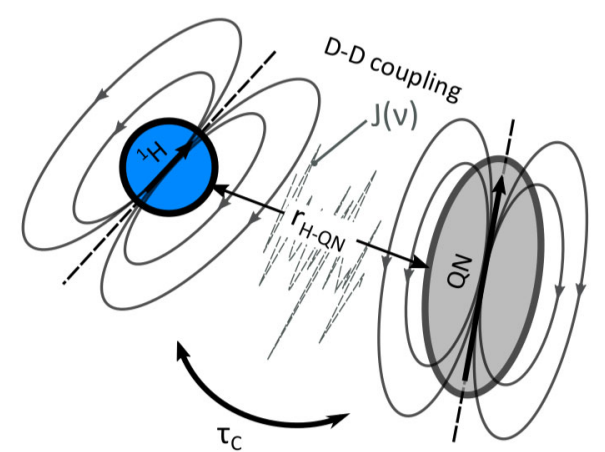

(a)

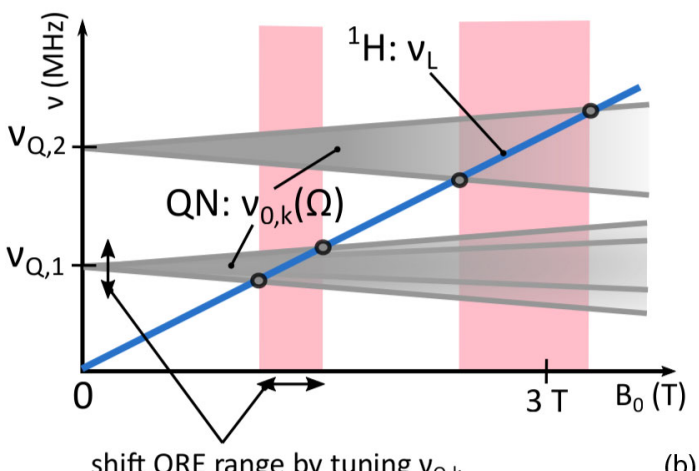

(b)

FIG. 2. (a) The schematic principle of QRE in protons due to the interaction with close-by $\mathrm{QN}$ via magnetic D-D coupling. (b) The transition frequencies of both spin ensembles, $\nu_{L}$ for the protons (blue line) and $\nu_{0, k}(\Omega)$ for the QNs (grey), depending on an externally applied flux density $B_{0}$. Where transitions of both spin ensembles match, QRE can be expected as for the considered case at $3 \mathrm{~T}$. This area is indicated by the vertical red bands. Panel (b) also gives an impression of how the QRE peaks are shifted by tuning the pure quadrupole frequency.

timescale and model of the motional dynamics (assuming an exponentially decaying correlation time function). The first condition arises because the D-D interaction strength scales with the distance $r_{\mathrm{H}-\mathrm{QN}}$ between the proton and the QN as $1 / r^{6}$. The second condition implies that the correlation time $\tau_{C}$ of the spin fluctuations has to be long enough to fulfill the condition $2 \pi \nu_{Q, k} \tau_{C}>1$.

Subsequently, we will focus on the resonance-condition for QRE, which can be written as

$$
\nu_{L}=\nu_{0, k}(\Omega)
$$

where $\nu_{L}=B_{0} \gamma_{H}$ is the Larmor frequency of the protons, and $\nu_{0, k}(\Omega)$ one of the allowed transition frequencies $k$ of the $\mathrm{QN}$, which result from transitions between the energy levels of the total QN Hamiltonian:

$$
H_{0}(I)(\Omega)=H_{Q}(I)(\Omega)+H_{Z}(I) .
$$

$H_{Q}$ is the pure quadrupole Hamiltonian, $H_{z}$ is the Zeeman Hamiltonian (see Appendix A), and $I$ is the nuclear spin of the $\mathrm{QN}, I>1 / 2$.
According to Eq. (1), the transition crossings occur only at particular flux densities, which is one reason for the frequency selectivity of QRE. This fact is also reflected in Fig. 2(b) (which is just another viewpoint of the situation in Fig. 1), where the qualitative transition patterns of both spin ensembles are shown in dependence on $B_{0}$. The pure quadrupole transitions $\nu_{Q, k}$ form transition frequency bands $\nu_{0, k}(\Omega)$ with increasing $B_{0}$. The bands are due to the fluctuations of the angle $\Omega$ between the EFG and the external field $B_{0}$ caused by molecular rotation. For solid powders, all intensity-weighted angles have to be considered. Crossings between the proton Larmor frequency $\nu_{L}$ and the QN-transition bands fulfill the resonance condition and open the possibility for QRE in protons. These regions (highlighted in red) can be shifted to a desired flux density, e.g., 3 T, by tuning the pure NQR spectrum [Fig. 2(b)]. To accomplish this in a rational manner, a sound understanding of the mechanisms involved in shifting the pure NQR pattern, in particular at the human body core temperature, is of major importance for the application of QRE in MRI CAs.

For convenience, in the following sections and tables, pure NQR transitions $\nu_{Q, k}$ are labeled consecutively from transition 1 to transition 4 , starting from the lowest $|1 / 2\rangle-|3 / 2\rangle$ to the highest $|7 / 2\rangle-|9 / 2\rangle \Delta m_{I}=1$ coherence. It is noted that, as soon as $\eta \neq 0$ or the Zeeman field points into an arbitrary direction with respect to the EFG, the states $|m\rangle$ are no longer pure Zeeman states and labeling according to $m_{I}$ becomes impracticable.

\section{A. QRE in solids and liquids}

Although the description of QRE on protons given above is quite general, the aggregation state (solid or liquid) as well as the exact proton pool (intra- or intermolecular) of the considered system has to be clearly defined and distinguished. An important difference is the source of D-D fluctuations responsible for the transfer of magnetization. Whereas, in solids, the spectral density of the fluctuations may be due to bond vibrations, for solutions containing both protons and QRE-based CA particles, diffusion and rotation are the main sources [compare Fig. 2(a)]. To simulate the relaxation dynamics of QRE in the liquid state, the stochastic Liouville approach $[23,34,35]$ is necessary, as the dynamical conditions for the Redfield theory [36,37], as used in solids, are not fulfilled anymore. Both theories necessarily require the knowledge of the NQR parameters $Q_{c c}$ and $\eta$. The most direct way for the determination of these parameters is zero-field NQRS in solid powders. For stochastic Liouville simulations in liquids, however, these parameters are only approximately valid, because they differ slightly [38] between free molecules in solution and molecules bound in a solid crystal. This issue will be discussed in Sec. III C. Beyond that, the QRE strength and position depends not only on the transition frequency match, but also on several 
additional parameters, such as the correlation time of the D-D fluctuations, the water exchange rate, and the relaxation behavior of the quadrupole nuclei. In the liquid state, there is no simple relationship, such as that illustrated in Figs. 1 and 2, which allows predicting QRE peaks directly from the NQR parameters, but relative changes of $Q_{c c}$ and $\eta$ are expected to lead to very similar relative shifts of the QRE peaks.

\section{RESULTS AND DISCUSSION}

\section{A. Compound selection}

As a group of model systems, we chose Bi-aryl compounds (in the form of crystalline powders) because of several practical reasons: Their most simple representative, triphenylbismuth, is commercially available and possesses a versatile and comparatively simple organic chemistry with the possibility of incorporating or grafting it onto nanoparticles for blood stream transportation. Moreover, at least partially complete NQR data sets are available for several Bi-aryl molecules [39], and these qualify some of them to be promising for QRE because they possess NQR transitions close to the frequencies that are required at clinical field strengths, i.e., 1.5 and $3 \mathrm{~T}$.

The structures of the compounds are shown in Fig. 3 and are labeled accordingly throughout the paper. The compounds were synthesized according to established procedures (see Appendix C, Table V). In total, eight compounds including triphenylbismuth (1) were investigated: tris(4-fluorophenyl) bismuth (2), tris(4dimethylaminophenyl) bismuth (3), tris(4-methoxyphenyl) bismuth (4), tris(2-methoxyphenyl) bismuth (5), tris(2-6dimethoxyphenyl) bismuth (6), triphenylbismuth dichloride (7), and fully deuterated triphenylbismuth (8). For samples 5 and 7, we identified two superimposed spectra corresponding to different $\mathrm{Bi}$ sites in the crystal $(5 \mathrm{~A}, 5 \mathrm{~B}$ and $7 \mathrm{~A}, 7 \mathrm{~B}$, respectively).

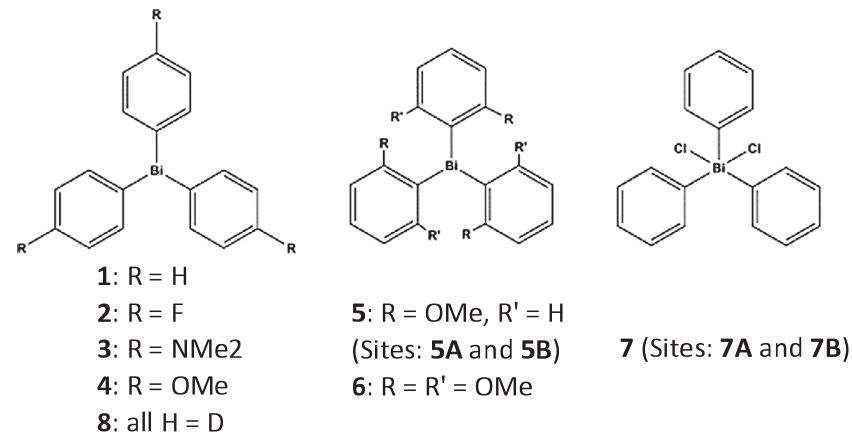

FIG. 3. Overview of Bi-aryl compounds and their structures: triphenylbismuth (1), tris(4-fluorophenyl) bismuth (2), tris(4dimethylaminophenyl) bismuth (3), tris(4-methoxyphenyl) bismuth (4), tris(2-methoxyphenyl) bismuth (5) (5A and 5B), tris(2-6-dimethoxyphenyl) bismuth (6), triphenylbismuth dichloride (7) (7A and 7B), and fully deuterated triphenylbismuth (8).
The tricoordinate $\mathrm{Bi}(\mathrm{III})$ compounds $1-6$ and 8 feature a trigonal pyramidal structure around $\mathrm{Bi}$ with sums of angles $\Sigma \mathrm{C}$-Bi-C between $280^{\circ}$ and $296^{\circ}$, where the phenyl rings adopt a tilted conformation with respect to the principal axis of the molecule (see Fig. 7 and Appendix C, Table V). Compound 7, triphenylbismuth dichloride, exhibits a pentacoordinate $\mathrm{Bi}(\mathrm{V})$ center, adopting a trigonal bipyramidal configuration, with $\mathrm{Cl}$ in apical position trans to each other, while the ipso-carbon atoms of the phenyl rings form the trigonal base and are in plane with the $\mathrm{Bi}$ atom. The phenyl rings are again tilted with respect to the $\mathrm{Cl}-\mathrm{Bi}-\mathrm{Cl}$ vector. Both structure types show almost perfect cylinder symmetry with respect to the pyramid's height axis. Basic structural data as well as structure references of the Bi-aryl compounds are summarized in Appendix C, Table V.

A rather important selection criterion already at this stage of development is the potential toxicity of the presented molecules. Bismuth compounds are used in therapeutic treatment of gastrointestinal disorders, suggesting a relatively low toxic potential after oral administration [40], with Peptobismol being the most prominent example. Although there are a few known cases, where bismuth compounds showed negative side effects (e.g., bismuth subgallate and bismuth tryglycollamate [41]), in general, bismuth compounds are not considered to feature a high degree of toxicity [24]. Particularly, arylbismuth compounds with stable $\mathrm{Bi}-\mathrm{C}$ bonds have not been reported to have high cytotoxic potential. For instance, triphenylbismuth (1), 1-phenyl-2-trimethlsilyl-1-benzostilyl-1benzobismepine, tris(4-methylphenyl) bismuth, and tris [2-(methoxymethyl)phenyl] bismuth, did not show cytotoxicity at $10 \mu \mathrm{M}$ [42]. However, establishing Bi compounds for intravenous application certainly requires further studies to assess their cytotoxicity also at higher concentrations as, e.g., used in conventional Gd-CAs (up to several $\mathrm{mM}$, which corresponds to about $0.1 \mathrm{mmol} / \mathrm{kg}$ body weight, according to the Gadovist product monograph). Promising data in this respect can be found in a study of dextran-coated bismuth-iron oxide nanohybrid contrast agents [29]. In this publication, in vivo experiments on mice showed extraction of the degradation products of the $\mathrm{CA}$ via kidneys and urine. The experiments were carried out with a dosage of $350 \mathrm{mg}$ Bi per kilogram body weight, i.e., more than $1.6 \mathrm{mmol} / \mathrm{kg}$ body weight. The investigated particles have been identified as being biocompatible and biodegradable.

\section{B. NQR spectroscopy}

From the eight different crystalline Bi-aryl powder samples introduced above, NQR parameters $Q_{c c}$ and $\eta$, relaxation times $T_{1, Q}$ and $T_{2, Q}$, and the linewidth of all accessible transitions were determined and are presented in Appendix C, Tables VI and VII. Every sample was characterized at $37^{\circ} \mathrm{C}$; for some of them, measurements were 
also carried out at the temperature of liquid nitrogen and room temperature (RT). To our knowledge, NQR data have not yet been published for tris(4-dimethylamino) bismuth (3), tris(2-methoxyphenyl) bismuth (4), tris(4-methoxyphenyl) bismuth (5), tris(2-6-dimethoxyphenyl) bismuth (6), and deuterated triphenylbismuth (8). Literature data were confirmed for the transition frequencies determined for triphenylbismuth (1) at RT and $77 \mathrm{~K}$ by Robinson et al. [43] and Weaver and Robinson [44], respectively, and for triphenylbismuth dichloride (7) by Brill and Long [45] at $300 \mathrm{~K}$, as well as for tris(4-fluorophenyl) bismuth (2) at $300 \mathrm{~K}$ by van der Kelen and Ketelaere [46].

\section{NQR spectrum and target frequency}

The collected data demonstrate the tuning possibilities of the quadrupole transition frequency by attaching different substituents. Figure 4 illustrates the positions of the transition frequencies of each sample measured at $37^{\circ} \mathrm{C}$ (colored dots) and RT (dots in brackets), respectively. In the case that a particular transition could not be measured with the current experimental setup, its frequency was calculated using Eq. (A7) of Appendix A and plotted as a grey square. Figure 4 shows also a grey-shaded area that highlights the target frequency range for the pure NQR peaks to create a QRE peak at a flux density range defined by a hypothetical FFC-MRI scanner (e.g., a standard medical system with nominal static flux density $B_{0}$ of $1.5 \mathrm{~T}$ or $2.89 \mathrm{~T}$, equipped with an additional insert coil which allows for shifting the flux density by $\pm 200 \mathrm{mT}$ ). Given that case, all samples have a transition within the required range (see Fig. 4). This field shift was chosen because it is technically feasible as shown by the $B_{0}$ insert coils used by Lee et al. [47] and Harris et al.

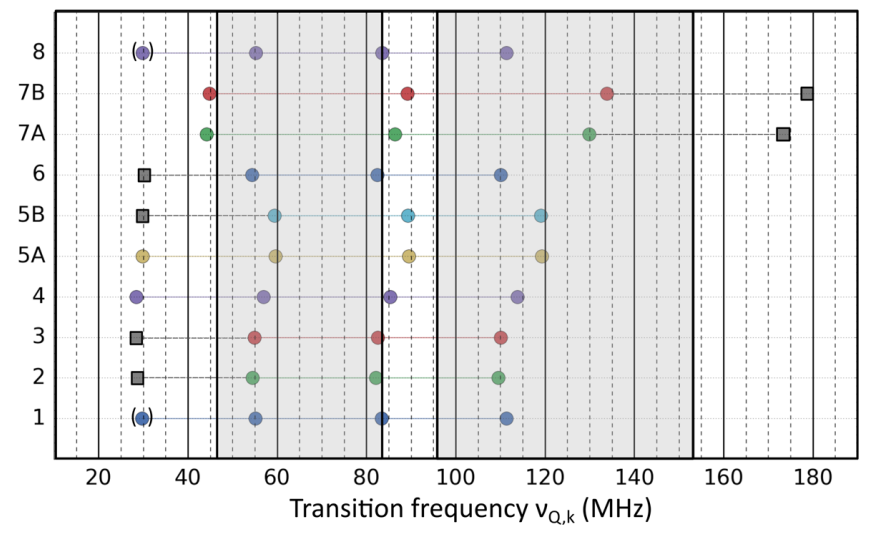

FIG. 4. NQR frequencies of samples 1-8. The pure NQR target frequency to create a QRE peak accessible for a FFC-MRI scanner with a $B_{0}$ shift of $1.5 \mathrm{~T} \pm 200 \mathrm{mT}$ and $2.89 \mathrm{~T} \pm 200 \mathrm{mT}$ is marked in grey. This frequency range can be calculated using the resonance condition Eq. (1) and the transition frequency spectrum as in Appendix A, Eq. (A7) [also compare to Fig. 2(b)]. Colored circles have been measured; grey squares mark calculated values [from Eq. (A7) in Appendix A]. Dots in brackets are measurements at RT; the rest have been obtained at $37^{\circ} \mathrm{C}$.
[19]. Another FFC-MRI system has been reported working at a nominal flux density of $2.89 \mathrm{~T} \pm 100 \mathrm{mT}$ [48].

Of course, it must be kept in mind that a certain shift in transition frequency can be expected when dissolving the solid powders in a solvent or grafting them onto a nanoparticle (NP). There are two important factors influencing the $Q_{c c}$, namely, the structure and chemical environment of the compounds (see Sec. III C). Additionally, the D-D interaction between ${ }^{209} \mathrm{Bi}$ and ${ }^{1} \mathrm{H}$ nuclei, which transfers the magnetization during $\mathrm{QRE}$, is modulated by the relative motion (mainly rotation) between the $\mathrm{CA}$ and water protons [23]. These influences can lead to frequency shifts of the QRE peaks in liquid with respect to those in solids, depending on the rotational correlation time between the two spin species. The frequency range illustrated in Fig. 4 is therefore treated only as a guideline rather than a precise decision rule for preselecting promising compounds.

\section{Observed NQR parameter variation}

$Q_{c c}$ and $\eta$ can be calculated from at least two experimentally determined NQR peaks using Eq. (A7) of Appendix A. When triphenylbismuth is considered as the reference system $\left(Q_{c c}=668.3 \mathrm{MHz}\right.$ at $\left.37^{\circ} \mathrm{C}\right), Q_{c c}$ shifts of several $\mathrm{MHz}$ can be observed when adding particular substituents (see Fig. 5). The strongest impact is observed when changing from three to five coordinate species [by adding two $\mathrm{Cl}$ atoms $(\mathrm{QN}, I=3 / 2)$ directly to the $\mathrm{Bi}$ center], which leads to an increase in $Q_{c c}$ by about $400 \mathrm{MHz}$. More subtle changes can be realized when incorporating substituents at different positions on the phenyl rings. Then $Q_{c c}$ shifts from about $+47 \mathrm{MHz}$ (methoxy- group on the ortho position of the phenyl rings) to- $11 \mathrm{MHz}$ (F on the para position of the phenyl rings) can be observed. The asymmetry parameter $\eta$ on the contrary changes only slightly from sample to sample and ranges between 0 and 0.1 (see Appendix C, Table VI), since the cylinder-symmetric EFG around $\mathrm{Bi}$ is maintained for all compounds. Compound 5 has the highest $\eta$ with 0.105 , whereas all compounds containing mono-methoxyphenyl do not possess any asymmetry. All associated values, including the transition

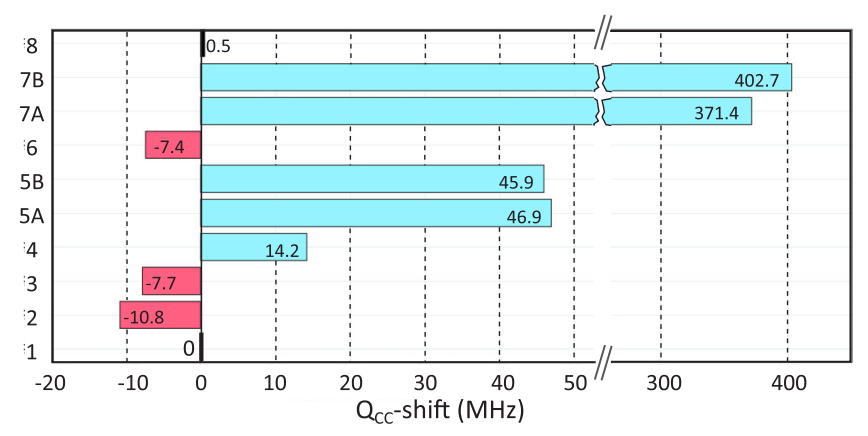

FIG. 5. $Q_{c c}$ shifts of Bi-aryl compounds with respect to triphenylbismuth (1) at $37^{\circ} \mathrm{C}\left(Q_{c c}=668.3 \mathrm{MHz}\right)$, depending on the ligand type, according to Fig. 3. 
frequencies for $37^{\circ} \mathrm{C}$, as well as some measurements at $77 \mathrm{~K}$, can be found in Appendix C, Table VI.

For samples 5 and 7, two superimposed ${ }^{209} \mathrm{Bi}$ NQR spectra, labeled A and B, have been observed. This is an indication for two different crystallographic sites within the crystal. In the case of triphenylbismuth dichloride, they correspond to crystallographically independent molecules on different lattice sites A and B [49]. For compound 5, Schuster et al. [50] reported two crystallographically independent molecules in the unit cell, where one is located on a threefold crystallographic axis (space group R3), while the second one is located on a general site within the unit cell with occupancies of 0.8802:0.1198. As the full width at half maximum (FWHM) of the peaks in spectrum $B$ is larger than in spectrum $A$ at all transitions (see Appendix C, Table VII), we assign site B as the disordered species. Noteworthy is the slight $Q_{c c}$ up-shift by $0.5 \mathrm{MHz}$ of deuterated triphenylbismuth (8) due to an isotope effect, which is not treated closely here.

\section{The influence of ligands}

As the EFG at the QN due to a point charge located at distance $r$ outside of the nucleus is proportional to $1 / r^{3}$ [30,51], only charge redistributions in the closest vicinity of the nucleus are relevant for shifting the transition frequency. Roughly, two influences on the EFG inducing the observed shifts can be distinguished: First, an additional substituent on whatever site will alter the electron density distribution of the whole molecule to a stronger or lesser degree due to valence orbital forming and charge polarization. This will, however, only have a considerable impact on the quadrupolar coupling strength if these changes affect the closer surrounding of the Bi center. For ligands at the outer position of the phenyl rings, this is expected to play only a minor role. Secondly, ligands introduce a change of both the molecular and the crystalline structure; this fact is in any case likely to have an impact on the EFG. In Fig. 6, this second influence is visualized by plotting the measured $Q_{c c}$ values versus the average $\mathrm{Bi}-\mathrm{C}$ bond length (see Appendix C, Table V) of each sample. The plot indicates a negative trend for $Q_{c c}$ when increasing the average $\mathrm{Bi}-\mathrm{C}$ distance within each molecule. Notably, tris(4-dimethylamino) bismuth (\#3) is a little bit off the trend. For this set of compounds, the plot in Fig. 6 may serve as a very rough estimation of what shift can be expected as soon as the structure change is known or vice versa.

\section{Temperature sensitivity}

The temperature dependency of the NQR peaks was investigated by temperature-swept NQRS experiments on selected samples. The temperature interval was selected with $+/-5^{\circ} \mathrm{C}$ around the core temperature of the human body, i.e., $37^{\circ} \mathrm{C}$. For reasons of simplicity, the temperature dependency of the transition frequencies is assumed to be linear in the observed range. The strongest measured

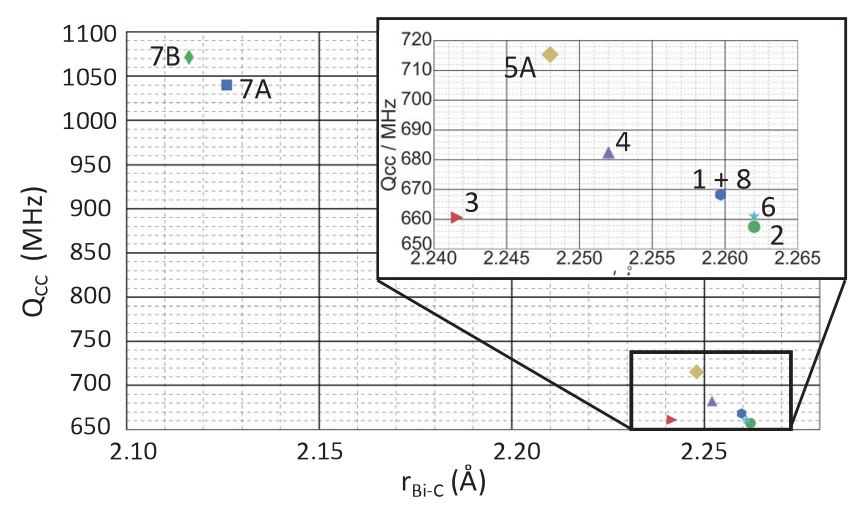

FIG. 6. $Q_{c c}$ versus average $\mathrm{Bi}-\mathrm{C}$ bond length. A trend for the investigated Bi-aryl compounds can be observed: The closer the $\mathrm{Bi}-\mathrm{C}$ distance, the higher $Q_{c c}$ is.

temperature coefficient is about $-10 \mathrm{kHz} / \mathrm{K}$ for transition 4 of compound 1, the weakest temperature coefficient of $-3 \mathrm{kHz} / \mathrm{K}$ was observed for transition 2 of compound $5 \mathrm{~A}$. The results are summarized in Appendix C, Table VIII. The frequency shift within $\pm 1^{\circ} \mathrm{C}$ is in general lower than a typical NQR-peak width (see Appendix C, Table VII) and is therefore not expected to play a major role in the application of the compounds as CA.

\section{Quadrupolar Relaxation}

Though the quadrupolar relaxation rates in the solid state are not of central interest for the scope of this paper, they have been tabulated for completeness because their determination formed part of our standard experimental procedure of the NQR characterization of the compounds. For almost all NQR transitions of the presented compounds, the relaxation times $T_{1, Q}$ and $T_{2, Q}$ were determined at $37^{\circ} \mathrm{C}$ sample temperature (see Appendix C, Table VII). This table forms a comprehensive database for deeper analysis in terms of relaxation theory, which is planned in follow-up papers. Moreover, it shows potential reasons why for some transitions the peaks could not be identified.

$T_{1, Q}$ ranges from as short as $50 \mu \mathrm{s}$ for transition 4 of sample 7 to as long as $3 \mathrm{~ms}$ for transition 1 of sample 1 . Also, for $T_{2}$, the range is wide and spans from about $30 \mu \mathrm{s}$ for sample 7, transition 4, up to $700 \mu$ s for sample 8, transition 4. $T_{2, Q}$ times somewhat lower than $30 \mu \mathrm{s}$ cannot be measured reliably with the current experimental setting. Thus, we conclude that some peaks could not be detected, because their $T_{2, Q}$ time is too short. In liquid nitrogen, the relaxation times usually increase considerably, thereby facilitating the identification of rapidly dephasing peaks.

\section{Quantum chemistry calculation}

$\mathrm{As}{ }^{209} \mathrm{Bi}$ is a rather heavy element, the $\mathrm{Bi}$-aryl compounds require careful considerations when performing quantum chemistry (QC) calculations of EFG tensors, especially when it comes to relativistic and electron correlation 
effects and their coupling [52-57]. In addition to the scalar relativistic (SR) effects, the influence of relativistic spinorbit coupling (SO) needs to be taken into account in the heavy element magnetic resonance parameters $[55,57,58]$. While combining relativistic theory with more elaborate electron correlation treatment with hybrid functionals of the density functional theory (DFT) is currently not feasible for periodic solids, we follow closely the approach of Roukala et al. for the nuclear shielding tensor of ${ }^{195} \mathrm{Pt}$ in molecular solids [59]. The following nomenclature is introduced to describe the calculation inputs: DFT Functional_Relativistic level_Basis Set, e.g., PBE_SO-ZORA_TZ2P, i.e., at spinorbit coupling zeroth order regular approximation (SOZORA) theory using the PBE DFT functional and TZ2P basis set, and it will be used to discuss the results.

\section{1. $Q_{c c}$ shift from a solid crystal to the $C A$}

Though the presented data have been gathered from solid crystalline samples, in the final application, the QRE effect will be used in CAs dispersed in liquids. These two states are expected to produce slightly different structures, as the molecules are integrated in different surroundings. The CA molecules are not embedded into a periodic crystal anymore, which causes what we call a "crystal effect" on the EFG. Also, the molecular structure itself is expected to relax (bond length and angles) in the altered surroundings, thus causing a "structure effect." As the particular surroundings of the CA are not exactly known at present, an isolated gas-phase structure is assumed as a reasonable approximation.

To figure out the role of these effects, three different cases have been considered at the PBE_SO-ZORA_TZ2P level for the reference system sample (1) (triphenylbismuth), which are shown in Fig. 7:

(i) case 1: molecule optimized in periodic surrounding + crystalline, periodic surrounding,

(ii) case 2: molecule optimized in periodic surrounding + isolated state,

(iii) case 3: molecule in isolated state (gas phase structure) + isolated state.

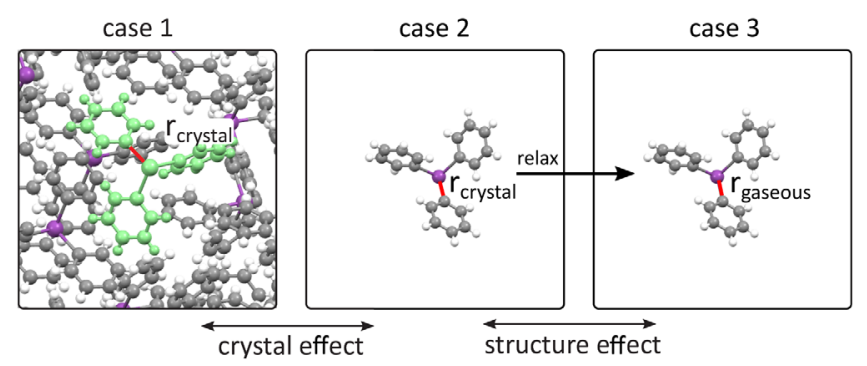

FIG. 7. Cases considered for QC calculations to estimate the crystal effect and the structure effect onto $Q_{C C}$. The average $\mathrm{Bi}-\mathrm{C}$ distance of the CASTEP-optimized crystal structure is $r_{\text {crystal }}=2.291 \AA$, while the distance is $r_{\text {gaseous }}=2.263 \AA$ in the gas phase [60] (see Appendix C, Table V). 3D models of the molecule structures are given in Supplemental Material [101].
The sole crystal effect makes up the difference between case 1 and 2, whereas the structure effect can be determined by taking the difference between case 2 and case 3.

Case 1 corresponds best to the experimental situation, except that the QC calculations were performed for static geometries neglecting thermal (vibrational) effects, whereas the experiment was carried out at $37^{\circ} \mathrm{C}$. Even though the data in Table I indicate that periodic calculations yield a rather good agreement with experiment, the comparison of absolute values must be treated with care, as there is room for improvement in both many-electron correlation and one-electron basis set treatments.

The PBE_SO-ZORA_TZ2P results in Table I show that, for $Q_{c c}$, the crystal effect of $+18.2 \%$ is much more important than the structure effect of $-2.7 \%$ due to the geometry change of the molecule from the gas phase to the solid phase. This means that when the triphenylbismuth molecule undergoes a transition from the solid crystalline to an isolated state, a $Q_{c c}$ shift of about $-15 \%$ can be expected. The asymmetry parameters $\eta$ are, in contrast to $Q_{c c}$, more sensitive to the change of local geometry around $\mathrm{Bi}$ than to, e.g., "global" crystal effects. The changes in $\eta$ are, therefore, not very strong, and the quadrupole tensor is practically cylindrically symmetric $(\eta \approx 0)$ in all cases.

\section{QC-parameter study}

The roles of different parameter improvements in QC calculations were tested in the case 2 for a single triphenylbismuth molecule. Table II shows the role of the improved QZ4P basis set, the better electronic correlation treatment with the hybrid PBE0 functional, and the impact of relativistic spin-orbit coupling effect at approximate SO-ZORA and exact two-component (X2C) relativistic theories, as well as at the fully relativistic four-component Dirac-Coulomb (DC) level of theory.

When turning from a TZ2P basis set to a QZ4P, $Q_{c c}$ changes by $+23.95 \mathrm{MHz}(4 \%)$ ( $\eta$ by +0.009$)$. This indicates that, with the lower basis set (TZ2P), the EFG has not fully converged yet, and QZ4P should be chosen if possible.

TABLE I. Comparison of QC results at the PBE_SO-ZORA_TZ2P level with experimental ones for $Q_{c c}$ and $\eta$ for triphenylbismuth in different surroundings.

\begin{tabular}{lcc}
\hline \hline Solid- CA comparison & $\eta(1)$ & $Q_{c c}(\mathrm{MHz})$ \\
\hline Case 1 & 0.049 & 661.1 \\
Case 2 & 0.059 & 539.5 \\
Case 3 & 0.001 & 557.3 \\
Experiment crystalline solid & 0.087 & 668.3 \\
Crystal effect (case 1-case 2) & -0.010 & 121.6 \\
Structure effect (case 2-case 3) & 0.058 & -17.9 \\
\hline \hline
\end{tabular}


TABLE II. Changes of $Q_{c c}$ and $\eta$ in a single triphenylbismuth molecule (case 2, if not noted otherwise) due to a better basis set (QZ4P with respect to TZ2P), hybrid PBE0 functional and relativistic spin-orbit coupling (SO-ZORA, X2C, and DC with respect to SR-ZORA, SF-X2C, and SF-DC, respectively). Nonrelativistic one- (NR) and four-component (LL) results are displayed for comparison.

\begin{tabular}{llcc}
\hline \hline Code & Level of theory (see Sec. VIB) & $\eta(1)$ & $Q_{c c}(\mathrm{MHz})$ \\
\hline ADF & PBE_SO-ZORA_TZ2P & 0.059 & 539.5 \\
& PBE_SO-ZORA_QZ4P & 0.069 & 563.4 \\
& PBE0_NR_QZ4P & 0.049 & 590.5 \\
& PBE0_SR-ZORA_QZ4P & 0.060 & 590.1 \\
& PBE0_SO-ZORA_QZ4P & 0.062 & 669.8 \\
& (crystal, case2) & & \\
& PBE0_SO-ZORA_QZ4P & 0.000 & 687.5 \\
& (gas, case3) & & \\
DIRAC & PBE0_LL_RPF-4Z (cc-pVDZ) & 0.048 & 587.9 \\
& PBE0_SF-DC_RPF-4Z (cc-pVDZ) & 0.053 & 645.1 \\
& PBE0_DC_RPF-4Z (cc-pVDZ) & 0.049 & 674.0 \\
PBE0_SF-X2C_RPF-4Z (cc-pVDZ) & 0.053 & 644.7 \\
PBE0_X2C_RPF-4Z (cc-pVDZ) & 0.049 & 673.2 \\
\hline \hline
\end{tabular}

A change from the pure GGA (PBE) to a hybrid DFT functional (PBE0), including $25 \%$ of the exact HartreeFock exchange, has a stronger influence on the $Q_{c c}$ value by an amount of $106.4 \mathrm{MHz}(19 \%)$ ( $\eta$ by -0.006$)$. According to Teodoro and Haiduke [56], hybrid DFT functionals provide reasonable but slightly underestimated EFG values for ${ }^{209} \mathrm{Bi}$ in diatomic molecules with respect to high-level $a b$ initio DC-CCSD results. The present magnitude increase due to $\mathrm{PBE} 0$ is most probably an improvement and, hence, the hybrid DFT functional is preferred for the estimation of $Q_{c c}$.

The rather large relativistic effect on $Q_{c c}$ of about $+80 \mathrm{MHz}(+14 \%$, with respect to PBE0_NR_QZ4P) associated with the heavy ${ }^{209} \mathrm{Bi}$ isotope is well described at the best PBE0_SO-ZORA_QZ4P level, including both $\mathrm{SR}$ and SO relativistic effects. This is confirmed by comparing it with the results from the fully relativistic four-component Dirac-Coulomb (PBE0_DC_RPF-4Z/ cc-pVDZ) and its much lighter exact two-component (PBE0_X2C_RPF-4Z/cc-pVDZ) approximation. While the ${ }^{209} \mathrm{Bi}$ basis set is presumably well converged in both cases, the small difference in $\eta$ is probably due to a different basis set of the ligands (TZ2P vs cc-pVDZ).

The seemingly quite large relativistic SO effect (PBE0_ SO-ZORA_QZ4P vs PBE0_SR-ZORA_QZ4P) on $Q_{c c}$, of about $+80 \mathrm{MHz}(+14 \%)$, is clearly overestimated by the ZORA method. The same effect at the fully relativistic $\mathrm{DC}$ and approximative $\mathrm{X} 2 \mathrm{C}$ level of theory is only about $+29 \mathrm{MHz}(+4 \%)$ when compared to results with the corresponding scalar relativistic SF-DC and SF-X2C results. Actually, the SR-ZORA value for $Q_{c c}$ is very similar with values of both nonrelativistic one- (NR) and four-component [Levy-Leblond (LL)] theories. Therefore, for ${ }^{209} \mathrm{Bi}$ in this complex, the so-called ZORA-4 scalar relativistic approximation, including a small-component density "picture-change correction" [61] used in ADF, gives a very small scalar relativistic effect on EFG. This is an example of a somewhat arbitrary division of SR and SO relativistic effects by different methods [62]. Therefore, the preferable SR approximation for EFG is obtained with the Dyall's spin-free Hamiltonian [63] used in the SF-DC and SF-X2C calculations with DIRAC code, which provides reasonable estimation close to the fully relativistic value.

The methods of choice for the molecular modeling of $\mathrm{Bi}$ complexes would be PBE0_SO-ZORA_QZ4P or PBE0_ X2C_RPF-4Z/cc-pVDZ combinations that provide good approximations for the fully relativistic PBE0_DC_RPF4Z/cc-pVDZ calculation with much lower cost (e.g., X2C takes approximately a tenth of the time of the DC calculation). However, for the case study in Sec. III C 1, we use PBE_SO-ZORA_TZ2P, as for the treatment of a periodic surrounding (ADF-BAND code), no higher basis set is available.

The PBE0_SO-ZORA_QZ4P calculation gives a $Q_{c c}$ value of about $-17.65 \mathrm{MHz}(-2.6 \%)$ for the structure correction (see Table II, changes from case 3 to case 2). The same relative change was predicted at the much lighter PBE_SO-ZORA_TZ2P level $(-2.7 \%)$, as seen in Table I, even though the absolute values are much smaller there. This shows that relative changes can be studied at a less advanced method and theory level as well.

\section{SUMMARY AND CONCLUSION}

The experimental investigation of eight different Bi-aryl compounds by means of NQR spectroscopy is an important step towards the rational synthesis of QRE-based CAs. The acquired data represent a compendium of promising $\mathrm{Bi}$ compounds with their NQR parameters $Q_{c c}$ and $\eta$, quadrupole transition frequencies, and associated relaxation times $T_{1, Q}$ and $T_{2, Q}$, mainly at the medically relevant body temperature of $37^{\circ} \mathrm{C}$. Depending on the type of ligand and its molecular position, frequency shifts of the quadrupole coupling constant $\left(Q_{c c}\right)$ from $-10.8 \mathrm{MHz}$ up to $400 \mathrm{MHz}$ with respect to triphenylbismuth $\left(Q_{c c}=\right.$ 668.3 MHz) have been observed, whereas the asymmetry parameter $\eta$ stays in a range of 0 to 0.1 . According to our analysis, this NQR parameter range is especially favorable for the utilization of QRE in clinical 1.5 or $3 \mathrm{~T}$ MRI scanners, provided they are equipped with a fast field cycling insert coil (FFC-MRI). The temperature dependence of the transition frequencies found at the human body core temperature (typically around $-10 \mathrm{kHz} / \mathrm{K}$ ) is not expected to affect the QRE contrast adversely. The average $\mathrm{Bi}-\mathrm{C}$ bond distance of each compound, which depends on ligand type and position, has been found to be correlated with $Q_{c c}$; when increasing the bond distance, a decrease in $Q_{c c}$ is observed (for a bond distance between 2.116 and 
$2.262 \AA, Q_{c c}$ decreases from 1071 to $675 \mathrm{MHz}$ ). From these ligand induced frequency shifts, one can also think of applications for QRE enhancement in fixed field scanners: An EFG change due to chemical interactions (e.g., in the context of ligand targeting) would "tune" or "detune" the QRE peak to or from the scanner frequency,, e.g., 1.5 T, thus producing contrast in the image.

As the transition frequencies cannot be expected to be exactly the same in the liquid state, it is of great interest to predict the expected discrepancies. In principle, this can be achieved by calculating the EFG modification with modern ab initio quantum chemistry (QC) methods. When modeling the transition of triphenylbismuth from a periodic molecular crystal to an isolated gas-phase molecule, our QC simulations predict a $Q_{c c}$ shift of $-18.2 \%$ due to the crystal effect and of $2.7 \%$ due to a compensating structural effect. These results suggest a possible downshift of the transition frequencies of about $15 \%$ when passing from solid to liquid.

Of course, the reliability of these findings depends on the validity of the model. In order to select the best QC settings, a parameter study has been performed. In particular, we face the problem of calculating electron distributions in the vicinity of high-mass, high-spin nuclei. It could be shown that the influence of relativistic effects, the chosen basis set, and the density functional are of great relevance; the resulting parameter set suggests PBE0_SO-ZORA_QZ4P with ADF code or PBE0_X2C_RPF-4Z/cc-pVDZ with DIRAC code. As an interesting byproduct, the generated NQRS database provides test data for the validation of EFG calculations with QC methods.

The strong variation of the $T_{1, Q}$ and $T_{2, Q}$ relaxation times for the different samples and NQR transitions point out the experimental difficulties of NQR spectroscopy: At $37^{\circ} \mathrm{C}, T_{1, Q}$ times ranging from $3 \mathrm{~ms}$ down to $60 \mu \mathrm{s}$ and $T_{2, Q}$ times from $700 \mu \mathrm{s}$ down to $40 \mu$ s could be observed, which, on the one hand can lead to very long measurement times and, on the other hand, challenge the ring down speed of the spectrometer. In several cases, measurements at low temperature $(77 \mathrm{~K})$ helped to increase the signal quality due to slower $T_{2, Q}$ relaxation and an increased population difference of the spin states. The relaxation data are particularly interesting for modeling molecular dynamics and testing relaxation models for high-spin systems in the solid state,, e.g., based on Redfield theory.

To summarize, the investigation of eight Bi-aryl compounds has identified them as promising candidates for use as QRE-based contrast agents. Because of their frequency selective character, the compounds may add interesting options in molecular MRI diagnostics in the future. Three of the above investigated solid samples, namely tris(2methoxyphenyl) bismuth (5), tris(2-6-dimethoxyphenyl) bismuth (6), and triphenylbismuth dichloride (7), have already shown pronounced QRE peaks in yet unpublished proton $T_{1}$ relaxometry data at the expected frequencies. Furthermore, the comparatively low toxicity, mostly good stability against hydrolysis and versatile as well as simple chemistry, qualifies Bi-aryl compounds as fruitful research objects in the context of MRI contrast agents.

\section{OUTLOOK}

Though the discussion of QRE in solids is out of the scope of this paper, we would like to mention that pronounced QRE peaks have already been observed. As an example, in Fig. 8 the $T_{1}$-NMRD profile of solid, crystalline tris(2methoxyphenyl) bismuth (5) is presented, which shows pronounced QRE peaks from inherent protons. These findings are not primarily relevant for the envisaged CAs; nevertheless, the data are highly motivating for undertaking the next logical steps: grafting the abovementioned core compounds onto NPs and solubilizing them to act as carriers through the bloodstream. The contrast enhancing effect can then be investigated also on free solvent protons of the system ${ }^{1} \mathrm{H}$ containing solvent $+\mathrm{NP}+\mathrm{CA}$ core compound by relaxation measurements. In particular, questions concerning the size and structure of the carrier particles can then be addressed to shape dynamical conditions and water exchange for optimizing D-D coupling and, thus, the magnetization transfer efficiency of the QRE. Another key parameter is the ${ }^{1} \mathrm{H}-\mathrm{QN}$ distance during closest approach. However, these questions need to be resolved before the expected increase in relaxivity of protons due to QRE CAs can be reasonably estimated.

From our current understanding, there are four points that should be focused on in future research in some more detail: Firstly, it can be concluded from the NMRD data in Fig. 8, as well as from theory, that the ${ }^{1} \mathrm{H}-\mathrm{QN}$ distance is of great importance for the D-D interaction $\left(1 / r^{6}\right)$. An average ${ }^{1} \mathrm{H}-\mathrm{QN}$ distance of about 3-4 $\AA$ is estimated for the solid,

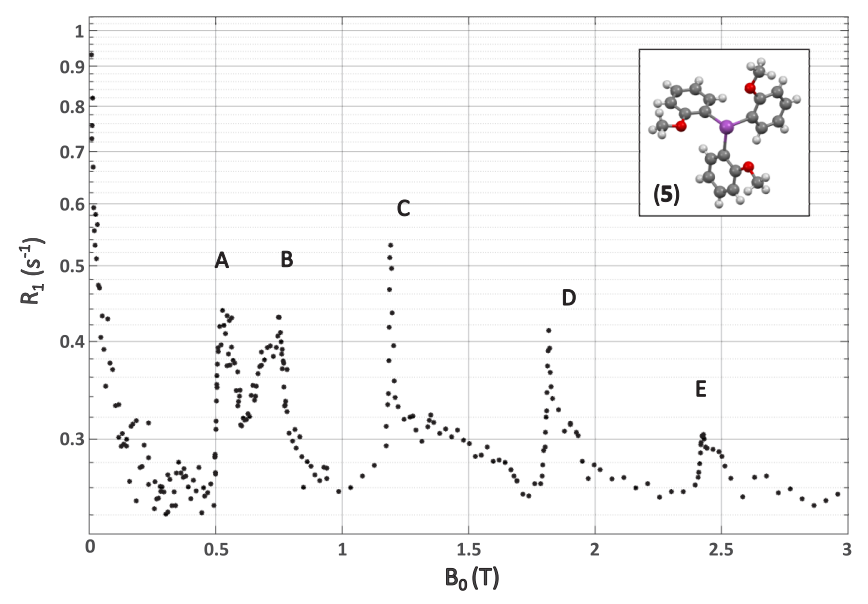

FIG. 8. Proton $T_{1}$-NMRD profile of the solid powder-sample (5) at $298 \mathrm{~K}$ obtained by a STELAR (Mede, Italy) Spinmaster relaxometer equipped with a magnet operating up to 3 T. Features A-E of the proton $R_{1}$ relaxation dispersion can be attributed unambiguously to QRE peaks superimposed on a background due to proton-proton dipolar coupling. 
which can be, at best, also assumed for solution protons exchanging with the coordination sphere of paramagnetic CAs (compare Gd-CAs with about 2.5-4 $\AA$ [64]). In this context, we expect approximately the same order of magnitude as in paramagnetic CAs. The second important parameter is the water exchange rate, which is expected to increase the enhancement per QN considerably when passing from solid to liquid. So far, there are no data available about this parameter in Bi-aryl compounds, which should be investigated also for a possible NP. The third parameter is the gyromagnetic ratio of the $\mathrm{QN}$, which is a lot smaller than that of the electrons in paramagnetic CAs. In this aspect, QRE CAs are clearly inferior. However, on the other hand, the relaxation enhancement seen with paramagnets is known to remain far below the maximum theoretical value because of the fast relaxation of the electrons. Here, we find a clear advantage in the QN compounds because the quadrupolar relaxation is usually much slower than that of electrons. This fourth parameter is, thus, probably essential for making QRE agents competitive.

In future QC studies, a refinement of the molecular surrounding of the CA can be considered. Furthermore, calculations to predict the EFG change of Bi-aryl compounds upon chemical modifications, i.e., different ligand types, can be envisaged. This could improve the CA-design procedure significantly by reducing the experimental workload.

\section{METHODS}

\section{A. NQR spectroscopy}

By means of NQR spectroscopy, the relaxation of a nuclear spin ensemble to the equilibrium distribution can be detected using a tank circuit. The most common NQR instruments are pulse-type FFT spectrometers $[32,65]$ that excite spin states using an RF-pulse sequence, comparable to standard NMR sequences but without exposing the sample to an external static flux density. For our investigations, we mainly use well-known sequences as, for example, spin echo (SE), free induction decay (FID), and inversion recovery (IR), using rectangular pulses together with phase cycling. Details concerning data analysis and fitting functions can be found in Appendix B, Eqs. (B1) and (B2).

Beyond characterizing QN spin systems, NQR spectroscopy has proven its scientific versatility in several applications and studies as, for example, research on molecular structure [66,67], superconductors [68], semiconductors [69], and phase transitions [70,71], as well as quantum computing [72] and the authentication of medicines [73].

Experiments were done with two pulse-type NQR spectrometers working with two complementary frequency sweep methods, both using a variety of transmit/receive coils. The self-built Graz-NQRS-MK1 [74] is particularly suitable for fast wide band sweeps up to 30-MHz bandwidth, whereas the commercially available Scout (Tecmag, Inc., USA) allows for advanced pulse sequence programming.
The probe coil is driven during transmit by a $500-\mathrm{W}$ amplifier and enables $\pi / 2$ pulses down to $300 \mathrm{~ns}$. In combination, the two systems cover a frequency range of $20 \mathrm{MHz}$ up to $150 \mathrm{MHz}$, with an available temperature range from liquid nitrogen (special cryo coils [75]) up to $50^{\circ} \mathrm{C}$ (dry-air flow thermostatization). The temperature is measured using a type $\mathrm{K}$ thermocouple close to the probe coil. For transportation, storage, and measurement, the samples are stored in glass vials of $10-\mathrm{mm}$ diameter and $40-\mathrm{mm}$ length in the form of a crystalline, solid powder.

\section{B. QC CALCULATION}

First, the ionic positions in a periodic crystal model were optimized at the DFT level using CASTEP [76] code and keeping lattice parameters in their experimental values of the starting geometry [77]. The PBE functional with TS dispersion correction [78] was used with ultrasoft pseudopotentials [79], a cutoff energy of $240 \mathrm{eV}$, and a MonkhorstPack $k$-point grid $4 \times 4 \times 1$ with spacing $<0.03 \AA^{-1}$ between points in each direction. After optimization, all forces acting on ions were less than $0.05 \mathrm{eV} / \AA$.

The EFG at the site of ${ }^{209} \mathrm{Bi}$ of triphenylbismuth was first computed for solid state structures with the periodic ADFBAND code of the Amsterdam Density Functional modeling suite [80-82] using the parameter-free PBE [83] DFT functional at the zeroth order regular approximation (ZORA) level of theory, including either SR (SR-ZORA) or both SR and SO effects (SO-ZORA). A more detailed study of electron correlation effects with a hybrid PBE0 $[84,85]$ functional was then carried out for a single triphenylbismuth molecule, either in its optimized solid state or experimentally found gas phase geometry [gas electron diffraction (GED)], by Berger et al. [60]. The relativistic effects on the EFG tensor were also studied at the fully relativistic four-component Dirac-Coulomb (DC) and the exact two-component $(\mathrm{X} 2 \mathrm{C})$ relativistic approximation, as well as their spin-free counterparts (SF-DC and SF-X2C), with the PBE0 functional using the DIRAC [86] code.

To estimate how much the EFG of the isolated molecule is affected due to geometry changes, we performed a structure optimization with ADF code at the PBED3_SR-ZORA_TZ2P level starting from the experimental gas phase structure [60]. The average changes in molecular geometry (see Table V in Appendix C) due to optimization caused a $1.2 \%$ decrease of $Q_{c c}(550.4 \mathrm{MHz}, \eta=0.001)$ at the PBE_SO-ZORA_TZ2P level of theory, which is a rather small change compared to other influences (see Tables I and II).

In the ADF-BAND calculations of EFG tensors, the Slater-type basis sets designed for ZORA calculations were used from the standard ADF basis set library [87]. Both triple- $\zeta$ with two (TZ2P) and quadruple- $\zeta$ with four (QZ4P) polarization functions basis sets were employed.

In calculations with DIRAC code, Gaussian-type nonrelativistic correlation-consistent polarized valence triple- $\zeta$ 
(cc-pVTZ) basis sets [88] were used for light $\mathrm{H}$ and $\mathrm{C}$ elements, whereas the relativistic prolapse-free quadruple- $\zeta$ (RPF-4Z) basis set [89] was used for ${ }^{209} \mathrm{Bi}$.

All the calculated nuclear quadrupole coupling constants $Q_{c c}$ were obtained from QC computed EFG tensors by using the most recent value for the nuclear quadrupole moment (NQM) of ${ }^{209} \mathrm{Bi}: Q\left({ }^{209} \mathrm{Bi}\right)=-415.1 \mathrm{mb}$ [57], which is within the error bars of the other recent determination of $Q\left({ }^{209} \mathrm{Bi}\right)=-420(8) \mathrm{mb}$ [56]. One should note that these values differ quite greatly from the old standard value of $Q\left({ }^{209} \mathrm{Bi}\right)=-516(15)[90]$.

\section{ACKNOWLEDGMENTS}

The authors wish to acknowledge the European Commission in the frame of the H2020 Programs CONQUER (FET-open) under Grant No. 665172, and EURELAX (COST-STSM) under Grant No. 1520937391 and the Academy of Finland (Project No. 285666) for financial support. Also, we want to acknowledge the CSC-IT Center for Science, Finland, for computational resources. P. L. acknowledges Professor Juha Vaara and Dr. Pär Håkansson for the scientific discussions. Also, we wish to thankfully acknowledge Dr. Eleonore Fröhlich for referencing and summarizing general questions on the toxicity of Bi compounds.

\section{APPENDIX A: THE NQR TRANSITION SPECTRUM}

There are basically two conditions that have to be met by the molecule under consideration to develop an observable nuclear spin transition spectrum: (1) A nucleus exhibiting a nonspherical charge distribution, so that its Taylor expansion gives a nonzero quadrupolar term (valid for nuclear spin $I>1 / 2$ ), and (2), a nonzero EFG generated by the charges external to the nucleus (derived from the spatial derivative of the corresponding electrostatic potential $\phi$ : $\phi_{i j}=\left[\left(\partial^{2} \phi\right) /\left(\partial x_{i} \partial x_{j}\right)\right]$, where $x_{i}, x_{j}$ are Cartesian coordinates $x, y, z$, and fulfilling $\Delta \phi=0)$.

The pure quadrupole Hamiltonian in some laboratory frame (LAB) can be expressed as the product of two irreducible rank two tensor operators $T_{m}^{(2)}$ and $V_{m}^{(2)}$ [37]:

$$
\begin{gathered}
H_{Q}^{\mathrm{LAB}}(I)=\frac{Q_{C C}}{I(2 I-1)} \sum_{m=-2}^{+2}-(1)^{m} T_{m}^{(2)} V_{-m}^{(2) \mathrm{LAB}}, \\
T_{0}^{(2)}(I)=\frac{1}{2}\left(3 \hat{I}_{z}^{2}-I(I+1)\right) \\
T_{ \pm 1}^{(2)}(I)=\frac{\sqrt{6}}{4}\left(\hat{I}_{z} \hat{I}_{ \pm}+\hat{I}_{ \pm} \hat{I}_{z}\right) \\
T_{ \pm 2}^{(2)}(I)=\frac{\sqrt{6}}{4} \hat{I}_{ \pm}^{2} .
\end{gathered}
$$

The product of the EFG strength eq $=\phi_{z z}$ and the quadrupole contribution $e Q$ is called the quadrupole coupling constant $Q_{c c}=e^{2} q Q / \hbar$. The $\hat{I}$ 's denote the usual spin operators.

In the principal axis system (PAS, where $\phi_{i j}=0$ for $i \neq j$ ), which is a reference frame bound to the molecule, the EFG tensor operator $V_{m}^{(2) P A S}$ can be written as

$$
\begin{aligned}
& V_{0}^{(2) \mathrm{PAS}}=\sqrt{\frac{3}{2}} \phi_{z z}=\sqrt{\frac{3}{2} \mathrm{eq}} \\
& V_{ \pm 1}^{(2) \mathrm{PAS}}=0, \quad V_{ \pm 2}^{(2) \mathrm{PAS}}=\frac{1}{2} \phi_{z z} \eta=\frac{1}{2} \mathrm{eq} \eta .
\end{aligned}
$$

$\eta=\left[\left(\phi_{x x}-\phi_{y y}\right) /\left(\phi_{z z}\right)\right]$ is the asymmetry parameter of the $\mathrm{EFG}$, ranges between 0 and 1 , and is a measure for the deviation from a cylindrically symmetric $(\eta=0)$ EFG.

When applying an external flux density $B_{0}$ to the spin system, the Zeeman Hamiltonian

$$
H_{Z}(I)=-\gamma_{\mathrm{QN}} B_{0} \hat{I}
$$

introduces an angle dependency $\Omega=(\alpha, \beta, \gamma)$ of the EFG with respect to the direction of $B_{0}$, which has to be considered when adding both quadrupolar and Zeeman interaction to the total Hamiltonian:

$$
H_{0}(I)(\Omega)=H_{Q}(I)(\Omega)+H_{Z}(I) .
$$

The tensor representation of $H_{Q}(I)$, Eq. (A1), allows rotating the quadrupole interaction by the Euler angles $\Omega=(\alpha, \beta, \gamma)$ into the main quantization axis of the total Hamiltonian, e.g., defined by $B_{0}$. The rotation from the PAS to a LAB system is performed using Wigner-D matrix elements:

$$
V_{m}^{(2) \mathrm{LAB}}(\Omega)=\sum_{m^{\prime}=-2}^{+2} D_{m^{\prime} m}^{(2)}(\Omega) V_{m^{\prime}}^{(2) \mathrm{PAS}} .
$$

The result of this transformation can be found in Possa et al. [72] and in Eq. (A9).

The corresponding transition frequencies $\nu_{0, k}$ (in the case of $B_{0}=0$, the pure $\mathrm{NQR}$ transition frequencies $\nu_{Q, k}$ ) can be calculated straightforwardly by diagonalizing the total Hamiltonian $H_{0}(I)$ and thereby obtaining the energy levels $E_{\alpha}$ of the spin system with respect to the eigenfunctions $\left\{\left|\psi_{\alpha}(\Omega)\right\rangle\right\}$ :

$$
\nu_{0, k}=E_{\alpha}-E_{\beta}=\left\langle\psi_{\alpha}\left|H_{0}(I)\right| \psi_{\alpha}\right\rangle-\left\langle\psi_{\beta}\left|H_{0}(I)\right| \psi_{\beta}\right\rangle .
$$

The eigenfunctions $\left\{\left|\psi_{\alpha}(\Omega)\right\rangle\right\}$ can be expressed as linear combinations of the functions $|m\rangle=\left|I, m_{I}\right\rangle$ of the Zeeman basis $\left\{\left|I, m_{I}\right\rangle\right\}$ with the magnetic quantum number $m_{I}=-I,-I+1, \ldots, I:\left|\psi_{\alpha}(\Omega)\right\rangle=\sum_{m=-I}^{2 I+1} a_{\alpha, m}(\Omega)|m\rangle$.

In the case of ${ }^{209} \mathrm{Bi}$, the nuclear spin of $I=9 / 2$ leads to four transitions with $\Delta m=1$ when $B_{0}=0$, as the energy levels are doubly degenerated in $m_{I}$. As soon as $B_{0} \neq 0$, the 
energy levels and, therefore, the transition frequencies split up and form a more complex pattern. The principal behavior of the NQR pattern depending on $B_{0}$ is shown in Figs. 1(a) and 1(b). In the case of a powder sample, an average across a spherical distribution of Euler angles can be calculated. The intensity of the spectrum in Fig. 1 is proportional to the Boltzmann population weighted transition probabilities:

$$
I\left(v_{Q, k}\right)=\left|\left\langle\psi_{\alpha}(\Omega)\left|H_{\mathrm{RF}}\right| \psi_{\beta}(\Omega)\right\rangle\right|^{2} \cdot \frac{\exp \left(-\frac{E_{\alpha}-E_{\beta}}{k_{B} T}\right)}{\sum_{i, j} \exp \left(-\frac{E_{i}-E_{j}}{k_{B} T}\right)} .
$$

$H_{\mathrm{RF}}$ is the radio frequency excitation Hamiltonian $H_{\mathrm{RF}} \sim \hat{I}_{\vec{x}} B_{\mathrm{RF}}$, where $\vec{x}$ is the direction of the transmit/receive coil generating an oscillating flux density $B_{\mathrm{RF}}$. T is the temperature and $k_{B}$ Boltzmann's constant.

In this work, only pure NQR transitions (no $B_{0}$ field) of powder samples are presented where the EFG is chosen to be in the PAS ( $\alpha=\beta=\gamma=0) . Q_{c c}$ and $\eta$ can be calculated numerically from two experimentally derived transition frequencies using Eq. (A7).

Performing the transformation according to Eq. (A6), the irreducible tensor operator elements have the form

$$
\begin{aligned}
& V_{0}^{(2) \mathrm{LAB}}=\frac{1}{2}\left[\frac{\left(3 \cos ^{2} \beta-1\right)}{2}+\frac{\eta}{4} \sin ^{2} \beta\left(e^{2 i \gamma}+e^{-2 i \gamma}\right)\right] \\
& V_{ \pm 1}^{(2) \mathrm{LAB}}=\frac{1}{2}\left[\mp \sqrt{\frac{3}{8}} \sin 2 \beta e^{ \pm i \alpha}+\frac{\eta}{\sqrt{6}}\left(-\frac{1 \mp \cos \beta}{2} \sin \beta e^{ \pm i(\alpha \mp 2 \gamma)}+\frac{1 \pm \cos \beta}{2} \sin \beta e^{i( \pm \alpha+2 \gamma)}\right)\right] \\
& V_{ \pm 2}^{(2) \mathrm{LAB}}=\frac{1}{2}\left[\sqrt{\frac{3}{8}} \sin ^{2} \beta e^{ \pm 2 i \alpha}+\frac{\eta}{\sqrt{6}}\left(\frac{(1 \mp \cos \beta)^{2}}{4} e^{ \pm 2 i(\alpha \mp \gamma)}+\frac{(1 \pm \cos \beta)^{2}}{4} e^{2 i( \pm \alpha+\gamma)}\right)\right] .
\end{aligned}
$$

The matrix representation of Eq. (A1) in the Zeeman basis $\left\{\left|I, m_{I}\right\rangle\right\}$, where $V_{m}^{(2) \mathrm{LAB}}(\Omega)$ has to be included from Eq. (A9), looks like

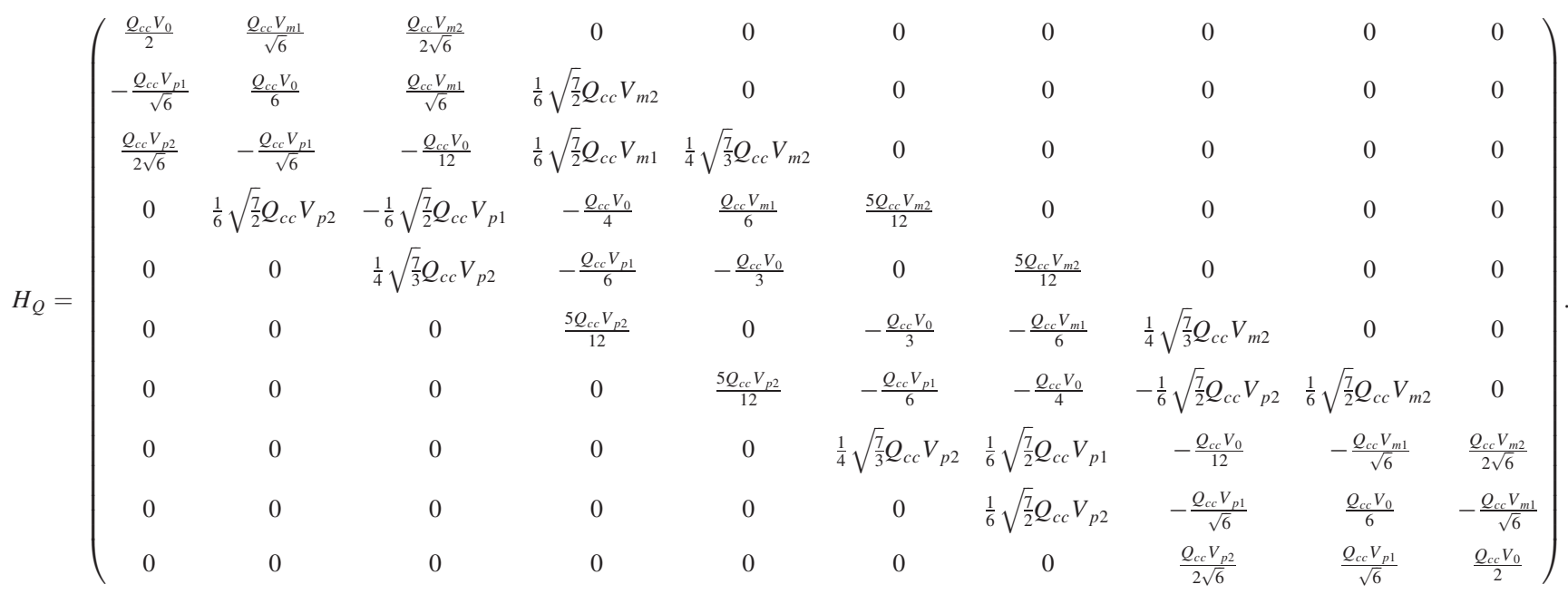

\section{APPENDIX B: DATA ANALYSIS AND FITTING PROCEDURE}

The spin-lattice $\left(T_{1, Q}\right)$ and spin-spin $\left(T_{2, Q}\right)$ relaxation times of all found transition peaks were experimentally determined using inversion recovery (IR) and spin echo (SE) sequences with variable inversion time $T_{I}$ and echo time $T_{E}$, respectively (for phase cycling, see Table III).
TABLE III. Phase of pulses for SE and IR.

\begin{tabular}{lcccc}
\hline \hline$\pi$ (only for IR) & $117^{\circ}$ & $117^{\circ}$ & $117^{\circ}$ & $117^{\circ}$ \\
\hline$\pi / 2$ & 0 & 1 & 2 & 3 \\
$\pi$ & 1 & 1 & 1 & 1 \\
$\mathrm{RX}$ & 0 & 3 & 2 & 1 \\
\hline \hline
\end{tabular}



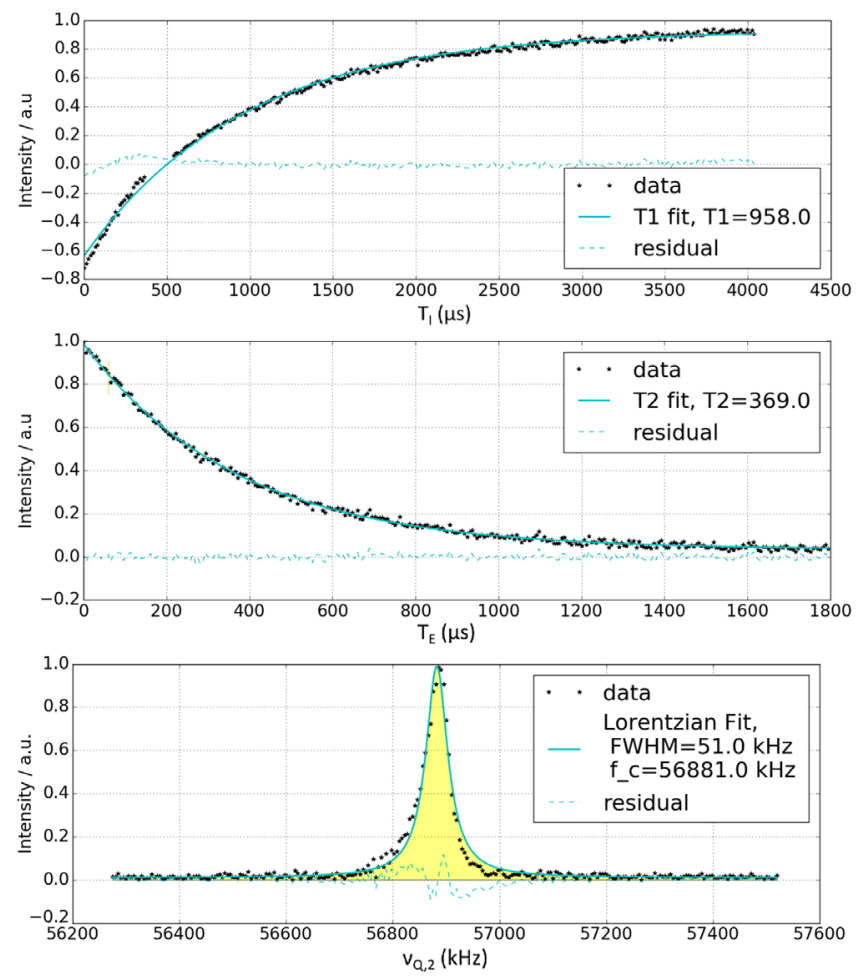

FIG. 9. Example for $T_{1}$ and $T_{2}$ measurement and fitted models; sample (4), transition 2 at $310 \mathrm{~K}$. For results, see Table IV.

The data were analyzed using the standard fitting procedure of the usual, well-known models [91]:

$$
\begin{gathered}
M_{\mathrm{IR}}\left(T_{I}\right)=M_{0}\left[1-2 \cdot \exp \left(-\frac{T_{I}}{T_{1, Q}}\right)\right]+B, \\
M_{\mathrm{SE}}\left(T_{E}\right)=M_{0} \exp \left(-\frac{T_{E}}{T_{2, Q}}\right)+B
\end{gathered}
$$

TABLE IV. Example for fitting result of Fig. 9, including result

\begin{tabular}{|c|c|c|c|c|}
\hline IR & $M_{0}($ arb.units $)$ & $T_{1, Q}(\mu \mathrm{s})$ & & $B$ (arb.units) \\
\hline Value & $7.8286 \times 10^{-1}$ & 958.3 & & $1.4471 \times 10^{-1}$ \\
\hline Std.error & $0.027 \times 10^{-1}$ & 7.8 & & $0.0371 \times 10^{-1}$ \\
\hline SE & $M_{0}$ (arb.units) & $T_{2, Q}(\mu \mathrm{s})$ & & $B$ (arb.units) \\
\hline Value & $9.5343 \times 10^{-1}$ & 368.6 & & $3.346 \times 10^{-2}$ \\
\hline Std.error & $0.0295 \times 10^{-1}$ & 2.5 & & $0.136 \times 10^{-2}$ \\
\hline $\mathrm{L}$ & $\gamma(\mathrm{kHz})$ & $\nu_{C}(\mathrm{kHz})$ & $I$ (arb.units) & $B$ (arb.units) \\
\hline Value & 25.38 & $56.882 \times 10^{3}$ & $9.63 \times 10^{-1}$ & $8.2 \times 10^{-3}$ \\
\hline Std.error & 0.50 & $0.000033 \times 10^{3}$ & $0.13 \times 10^{-1}$ & $1.7 \times 10^{-3}$ \\
\hline
\end{tabular}
and standard error according to Eqs. (B1), (B2), and (B3).

To fit the peak shape, a Lorentzian shape is assumed, which holds true for monoexponential decays:

$$
\begin{aligned}
L(\nu) & =\frac{I}{\left[1+\left(\frac{\nu-\nu_{C}}{\gamma}\right)^{2}\right]}+B \\
\text { FWHM } & =2 \gamma .
\end{aligned}
$$

An example of the application of the models is given in Fig. 9 and Table IV for tris(4-methoxyphenyl) bismuth (4), transition 2, at $310 \mathrm{~K}: T_{1, Q}=958 \mu \mathrm{s}, T_{2, Q}=369 \mu \mathrm{s}$, $\nu_{c}=56.88 \mathrm{kHz}, \mathrm{FWHM}=51 \mathrm{kHz}$, stepsize $\Delta T_{E}=4 \mu \mathrm{s}$, and $\Delta T_{I}=10 \mu \mathrm{s}$.

The temperature dependency of the transition frequency is assumed to be linear in the range of several degrees $\left(\Delta T+/-5^{\circ} \mathrm{C}\right)$ around the target temperature of $310 \mathrm{~K}$ $\left(37^{\circ} \mathrm{C}\right)$. The linear temperature coefficient $\mathrm{C}_{37}$ is determined by measuring the transition frequency $\nu_{Q, k}$ for several sample temperatures:

$$
\nu_{Q, k}(\mathrm{~T})=C_{37} \Delta T+\nu_{37}
$$

\begin{tabular}{|c|c|c|c|c|c|c|c|}
\hline $\mathrm{Nr}$ & Sample name & $\begin{array}{l}\text { Crystal } \\
\text { structure }\end{array}$ & $\begin{array}{l}\text { Average } \mathrm{Bi}-\mathrm{C} \\
\text { distance }(\AA)\end{array}$ & $\begin{array}{l}\text { Sum of angles } \\
\mathrm{C}-\mathrm{Bi}-\mathrm{C}\left({ }^{\circ}\right)\end{array}$ & $\begin{array}{l}\text { Structure } \\
\text { reference }\end{array}$ & $\begin{array}{l}\text { Hydrolytic } \\
\text { stability }\end{array}$ & $\begin{array}{l}\text { Synth. } \\
\text { reference }\end{array}$ \\
\hline 1 & Triphenylbismuth & Monoclinic C2/c & 2.260 & 281.68 & [77] & Yes & [92-94] \\
\hline 2 & $\begin{array}{l}\text { Tris(4-fluorophenyl) } \\
\text { bismuth }\end{array}$ & Monoclinic P2(1)/c & 2.262 & 285.38 & Not available & Yes & [94] \\
\hline 3 & $\begin{array}{l}\text { Tris(4-dimethylamino) } \\
\text { bismuth }\end{array}$ & Triclinic, P-1 & 2.242 & 283.04 & [95] & Yes & [96] \\
\hline 4 & $\begin{array}{l}\text { Tris(4-methoxyphenyl) } \\
\text { bismuth }\end{array}$ & Trigonal, R-3 & 2.252 & 281.37 & [94] & Yes & [94] \\
\hline
\end{tabular}

Raw data and fitting parameters for each measurement are available anytime in detail on request.

\section{APPENDIX C: EXPERIMENTAL DATA AND ERROR ESTIMATION}

TABLE V. Sample overview; crystal structures, structure info, references and source. 
TABLE V. (Continued)

\begin{tabular}{|c|c|c|c|c|c|c|c|}
\hline $\mathrm{Nr}$ & Sample name & $\begin{array}{l}\text { Crystal } \\
\text { structure }\end{array}$ & $\begin{array}{l}\text { Average } \mathrm{Bi}-\mathrm{C} \\
\text { distance }(\AA)\end{array}$ & $\begin{array}{l}\text { Sum of angles } \\
\mathrm{C}-\mathrm{Bi}-\mathrm{C}\left({ }^{\circ}\right)\end{array}$ & $\begin{array}{l}\text { Structure } \\
\text { reference }\end{array}$ & $\begin{array}{l}\text { Hydrolytic } \\
\text { stability }\end{array}$ & $\begin{array}{l}\text { Synth. } \\
\text { reference }\end{array}$ \\
\hline $5 \mathrm{~A}$ & $\begin{array}{l}\text { Tris(2-methoxyphenyl) } \\
\text { bismuth, site A }\end{array}$ & Rhombohedral R3 & 2.248 & $282.62 / 277.4$ & {$[50,97]$} & Yes & [98] \\
\hline $5 \mathrm{~B}$ & $\begin{array}{l}\text { Tris(2-methoxyphenyl) } \\
\text { bismuth, site B }\end{array}$ & Disordered & Not available & Not available & {$[50]$} & Yes & [98] \\
\hline 6 & $\begin{array}{l}\text { Tris(2-6- } \\
\text { dimethoxyphenyl) } \\
\text { bismuth }\end{array}$ & Monoclinic $\mathrm{P} 2{ }_{1} / \mathrm{c}$ & 2.262 & 296.8 & [99] & Yes & [98] \\
\hline $7 \mathrm{~A}$ & $\begin{array}{l}\text { Triphenylbismuth } \\
\text { dichloride, site A }\end{array}$ & $\begin{array}{l}\text { Orthorhombic } \\
\text { P2(1)2(1)2(1) }\end{array}$ & 2.129 & Not available & [49] & No & {$[93,100]$} \\
\hline $7 \mathrm{~B}$ & $\begin{array}{l}\text { Triphenylbismuth } \\
\text { dichloride, site B }\end{array}$ & $\begin{array}{l}\text { Orthorhombic } \\
\text { P2(1)2(1)2(1) }\end{array}$ & 2.116 & $\cdots$ & [49] & No & {$[93,100]$} \\
\hline 8 & $\begin{array}{l}\text { Triphenylbismuth } \\
\text { deuterated }\end{array}$ & Monoclinic $\mathrm{C} 2 / \mathrm{c}$ & See Nr.1 & See Nr.1 & Not available & Yes & Not available \\
\hline Not available & $\begin{array}{l}\text { Triphenylbismuth, } \\
\text { gas phase }\end{array}$ & Not available & 2.263 & 284.13 & [60], GED structure & Not available & Not available \\
\hline Not available & $\begin{array}{l}\text { Triphenylbismuth, } \\
\text { ADF optimized, } \\
\text { gas phase }\end{array}$ & Not available & 2.279 & 282.08 & Not available & Not available & Not available \\
\hline Not available & $\begin{array}{l}\text { Triphenylbismuth, } \\
\text { CASTEP optimized, } \\
\text { periodic }\end{array}$ & Monoclinic, $\mathrm{C} 2 / \mathrm{c}$ & 2.291 & 282.62 & Not available & Not available & Not available \\
\hline
\end{tabular}

TABLE VI. Measured NQR transition frequencies $\nu_{Q, k}$ and derived NQR parameter $Q_{c c}$ and $\eta$ at different temperatures $(310 \mathrm{~K}, 300 \mathrm{~K}$, and $77 \mathrm{~K}$ ) for eight Bi-aryl compounds.

\begin{tabular}{|c|c|c|c|c|c|c|c|c|}
\hline \multicolumn{9}{|c|}{ Transition frequency $\nu_{Q, k}(\mathrm{MHz})^{\mathrm{a}}$} \\
\hline Nr. & Sample name & Temp.(K) & 1 & 2 & 3 & 4 & $Q_{c c}(\mathrm{MHz})^{\mathrm{b}}$ & $\eta(1)^{\mathrm{c}}$ \\
\hline \multirow{3}{*}{1} & \multirow{3}{*}{ Triphenylbismuth } & 310 & 29.67 & 55.14 & 83.42 & 111.32 & 668.3 & 0.087 \\
\hline & & 300 & 29.76 & 55.21 & 83.50 & 111.42 & 668.9 & 0.083 \\
\hline & & 77 & 30.60 & 56.45 & 85.45 & 114.03 & 684.6 & 0.090 \\
\hline \multirow{2}{*}{2} & \multirow{2}{*}{ Tris(4-fluorophenyl) bismuth } & 310 & 28.60 & 54.51 & 82.11 & 109.54 & 657.5 & 0.071 \\
\hline & & 77 & 28.65 & 55.86 & 84.02 & 112.06 & 672.5 & 0.053 \\
\hline \multirow{2}{*}{3} & \multirow{2}{*}{ Tris(4- dimethylamino) bismuth } & 310 & 28.34 & 54.94 & 82.52 & 110.06 & 660.6 & 0.058 \\
\hline & & 77 & 28.23 & 56.20 & 84.35 & 112.47 & 674.8 & 0.020 \\
\hline \multirow{2}{*}{4} & \multirow{2}{*}{ Tris(4-methoxyphenyl) bismuth } & 310 & 28.49 & 56.88 & 85.34 & 113.77 & 682.5 & 0.000 \\
\hline & & 77 & 29.17 & $\mathrm{n} / \mathrm{a}$ & $\mathrm{n} / \mathrm{a}$ & $\mathrm{n} / \mathrm{a}$ & $\mathrm{n} / \mathrm{a}$ & $\mathrm{n} / \mathrm{a}$ \\
\hline $5 \mathrm{~A}$ & Tris(2-methoxyphenyl) bismuth site A & 310 & $(29.82)$ & 59.64 & 89.47 & 119.27 & 715.2 & 0.000 \\
\hline $5 \mathrm{~B}$ & Tris(2-methoxyphenyl) bismuth site B & 310 & 29.76 & 59.45 & 89.29 & 119.06 & 714.3 & 0.000 \\
\hline 6 & Tris(2-6-dimethoxyphenyl) bismuth & 310 & 30.14 & 54.40 & 82.45 & 110.06 & 660.9 & 0.105 \\
\hline \multirow{2}{*}{$7 \mathrm{~A}$} & \multirow{2}{*}{ Triphenylbismuth dichloride site A } & 310 & $(44.14)$ & 86.40 & 129.91 & 173.30 & 1039.7 & 0.046 \\
\hline & & 77 & 45.51 & 87.50 & 131.80 & 175.80 & 1055.3 & 0.063 \\
\hline \multirow{2}{*}{$7 \mathrm{~B}$} & \multirow{2}{*}{ Triphenylbismuth dichloride site B } & 310 & $(44.81)$ & 89.20 & 133.86 & 178.50 & 1071.0 & 0.020 \\
\hline & & 77 & 45.80 & 91.24 & 136.90 & 182.60 & 1095.4 & 0.020 \\
\hline \multirow{2}{*}{8} & \multirow{2}{*}{ Triphenylbismuth deuterated } & 310 & 29.68 & 55.20 & 83.49 & 111.40 & 668.8 & 0.086 \\
\hline & & 300 & 29.82 & 55.25 & 83.58 & 111.56 & 669.9 & 0.098 \\
\hline \multicolumn{9}{|c|}{$\begin{array}{l}\text { n/a., not available; red, italic values indicate values that have been theoretically predicted and have not been measured yet; values in } \\
\text { brackets have larger errors. } \\
\text { a } \Delta v_{Q, k}=+/-10 \mathrm{kHz}(310 \mathrm{~K}), \Delta v_{Q, k}=+/-30 \mathrm{kHz}(300 \mathrm{~K} \text { and } 310 \mathrm{~K} \text { in brackets }), \Delta v_{Q, k}=+/-5 \mathrm{kHz}(77 \mathrm{~K}) \\
\mathrm{b} \Delta Q_{c c}=+/-0.4 \mathrm{MHz}(310 \mathrm{~K}), \Delta Q_{c c}=+/-0.6 \mathrm{MHz}(300 \mathrm{~K} \text { and } 310 \mathrm{~K} \text { in brackets }), \Delta Q_{c c}=+/-0.2 \mathrm{MHz}(77 \mathrm{~K}) \\
{ }^{\mathrm{c}} \Delta \eta=+/-0.001(310 \mathrm{~K}), \Delta \eta=+/-0.004(300 \mathrm{~K} \text { and } 310 \mathrm{~K} \text { in brackets), } \Delta \eta=+/-0.001(77 \mathrm{~K})\end{array}$} \\
\hline
\end{tabular}


TABLE VII. Measured NQR relaxation times $T_{1, Q}$ and $T_{2, Q}$, FWHM of Lorentzian peak shape, and transition frequencies $\nu_{Q, k}$.

\begin{tabular}{|c|c|c|c|c|c|c|c|}
\hline Nr. & Sample name & Transition & Temperature $(\mathrm{K})^{\mathrm{a}}$ & $T_{1, Q}(\mu \mathrm{s})^{\mathrm{b}}$ & $T_{2, Q}(\mu \mathrm{s})^{\mathrm{c}}$ & $\mathrm{FWHM}(\mathrm{kHz})^{\mathrm{d}}$ & $\nu_{Q, k}(\mathrm{MHz})^{\mathrm{e}}$ \\
\hline \multirow[t]{11}{*}{1} & Triphenylbismuth & 2 & 310 & 1123 & 277 & 4 & 55.14 \\
\hline & & 3 & 310 & 548 & 436 & 15 & 83.42 \\
\hline & & 4 & 310 & 835 & 486 & 5 & 111.32 \\
\hline & & 1 & 300 & 2915 & 80 & 10 & 29.76 \\
\hline & & 2 & 300 & 1547 & 222 & 7 & 55.21 \\
\hline & & 3 & 300 & 820 & 396 & 7 & 83.50 \\
\hline & & 4 & 300 & 966 & 434 & 9 & 111.42 \\
\hline & & 1 & 77 & 63738 & 102 & 27 & 30.6 \\
\hline & & 2 & 77 & 18000 & 394 & 12 & 56.45 \\
\hline & & 3 & 77 & 16400 & 802 & 4 & 85.45 \\
\hline & & 4 & 77 & 17029 & 822 & 5 & 114.03 \\
\hline \multirow[t]{7}{*}{2} & Tris(4-fluorophenyl) bismuth & 2 & 310 & 201 & 81 & 10 & 54.51 \\
\hline & & 3 & 310 & 91 & 66 & 18 & 82.11 \\
\hline & & 4 & 310 & 89 & 83 & 22 & 109.54 \\
\hline & & 1 & 77 & 11182 & 48 & 22 & 28.65 \\
\hline & & 2 & 77 & 2930 & 450 & 8 & 55.86 \\
\hline & & 3 & 77 & 2322 & 581 & 9 & 84.02 \\
\hline & & 4 & 77 & 1754 & 546 & 10 & 112.06 \\
\hline \multirow[t]{5}{*}{3} & Tris(4- dimethylamino) bismuth & 2 & 310 & 238 & 109 & 19 & 54.94 \\
\hline & & 3 & 310 & 193 & 108 & 32 & 82.52 \\
\hline & & 4 & 310 & 231 & 115 & 20 & 110.06 \\
\hline & & 3 & 77 & 2600 & 360 & Poor fit & 84.348 \\
\hline & & 4 & 77 & 3700 & 400 & Poor fit & 112.469 \\
\hline \multirow[t]{5}{*}{4} & Tris(4-methoxyphenyl) bismuth & 1 & 310 & 2897 & 51 & 17 & 28.49 \\
\hline & & 2 & 310 & 958 & 369 & 51 & 56.88 \\
\hline & & 3 & 310 & 768 & 245 & 43 & 85.34 \\
\hline & & 4 & 310 & 872 & 294 & 66 & 113.77 \\
\hline & & 1 & 77 & $\mathrm{n} / \mathrm{a}$ & 42 & 14 & 29.12 \\
\hline \multirow[t]{4}{*}{$5 \mathrm{~A}$} & Tris(2-methoxyphenyl) bismuth & 1 & $(310)$ & 1261 & 57 & 11 & 29.82 \\
\hline & Site A & 2 & 310 & 570 & 370 & 25 & 59.64 \\
\hline & & 3 & 310 & 334 & 284 & 36 & 89.47 \\
\hline & & 4 & 310 & 426 & 315 & 41 & 119.27 \\
\hline \multirow[t]{3}{*}{$5 B$} & Tris(2-methoxyphenyl) bismuth & 2 & 310 & 64 & 58 & 48 & 59.45 \\
\hline & Site B & 3 & 310 & 64 & 51 & 45 & 89.3 \\
\hline & & 4 & 310 & 61 & 67 & 63 & 119.06 \\
\hline \multirow[t]{3}{*}{6} & Tris(2-6-dimethoxyphenyl) bismuth & 2 & 310 & 122 & 69 & 10 & 54.40 \\
\hline & & 3 & 310 & 50 & 51 & 14 & 82.45 \\
\hline & & 4 & 310 & 78 & 56 & 14 & 110.06 \\
\hline \multirow[t]{5}{*}{ 7A } & Triphenylbismuth dichloride & 1 & $(310)$ & 98 & 52 & Poor fit & 44.14 \\
\hline & Site A & 2 & 310 & 63 & 50 & 25 & 86.40 \\
\hline & & 3 & 310 & 46 & 44 & 36 & 129.91 \\
\hline & & 2 & 77 & 940 & 310 & 7 & 87.50 \\
\hline & & 1 & 77 & 1846 & 90 & 16 & 45.51 \\
\hline \multirow[t]{5}{*}{ 7B } & Triphenylbismuth dichloride & 1 & $(310)$ & 241 & 40 & 6 & 44.81 \\
\hline & Site B & 2 & 310 & 108 & 46 & 9 & 89.20 \\
\hline & & 3 & 310 & 54 & 28 & 17 & 133.86 \\
\hline & & 1 & 77 & 985 & 69 & Poor fit & 45.80 \\
\hline & & 2 & 77 & 397 & 311 & 8 & 91.24 \\
\hline \multirow[t]{7}{*}{8} & Triphenylbismuth deuterated & 1 & 300 & 2381 & 418 & 20 & 29.82 \\
\hline & & 2 & 300 & 1472 & 525 & 11 & 55.25 \\
\hline & & 3 & 300 & 909 & 636 & 13 & 83.58 \\
\hline & & 4 & 300 & 1049 & 693 & 16 & 111.56 \\
\hline & & 2 & 310 & 1104 & 621 & 11 & 55.20 \\
\hline & & 3 & 310 & 646 & 629 & 11 & 83.49 \\
\hline & & 4 & 310 & 725 & 712 & 15 & 111.40 \\
\hline
\end{tabular}

n/a, not available; "poor fit": due to poor frequency resolution, temperatures in brackets have higher uncertainty atemperature accuracy: $0.5 \mathrm{~K}$

${ }^{\text {b,c }}$ at $300 \mathrm{~K} / 310 \mathrm{~K}$ : For $T_{2, Q}>200 \mu \mathrm{s}, \Delta T_{1, Q}:<12 \%, \Delta T_{2, Q}:<12 \%$

$$
\text { For } T_{2, Q}<200 \mu \mathrm{s}, \Delta T_{1, Q}:<22 \%, \Delta T_{2, Q}:<22 \%
$$

b,c at $77 \mathrm{~K}$ : For $T_{2, Q}>200 \mu \mathrm{s}, \Delta T_{1, Q}:<7 \%, \Delta T_{2, Q}:<7 \%$

For $T_{2, Q}<200 \mu \mathrm{s}, \Delta T_{1, Q}:<12 \%, \Delta T_{2, Q}:<12 \%$

${ }^{\mathrm{d}} \Delta \mathrm{FWHM}:+/-1 \mathrm{kHz}$, due to fitting, see Table IV

${ }^{\mathrm{e}} \Delta \nu_{Q, k}$ at $310 \mathrm{~K}=+/-10 \mathrm{kHz}, \Delta \nu_{Q, k}$ at $(310 \mathrm{~K})=+/-30 \mathrm{kHz}, \Delta \nu_{Q, k}$ at $300 \mathrm{~K}=+/-30 \mathrm{kHz}$ 
TABLE VIII. Linear temperature coefficient $\mathrm{C}_{37}$ of selected NQR transition frequencies of several Bi-aryl compounds.

\begin{tabular}{|c|c|c|c|}
\hline Nr. & Sample name & Transition & $\mathrm{C}_{37}(\mathrm{kHz} / \mathrm{K})^{\mathrm{a}}$ \\
\hline \multirow[t]{2}{*}{1} & Triphenylbismuth & 3 & -9.2 \\
\hline & & 4 & -10.4 \\
\hline \multirow[t]{2}{*}{2} & Tris(4-flurophenyl) bismuth & 3 & -5.9 \\
\hline & & 4 & -6.4 \\
\hline \multirow[t]{2}{*}{3} & Tris(4-dimethylamino) bismuth & 3 & -5.7 \\
\hline & & 4 & -6.4 \\
\hline \multirow[t]{3}{*}{4} & Tris(4-methoxyphenyl) bismuth & 2 & -4.7 \\
\hline & & 3 & -6.2 \\
\hline & & 4 & -7.1 \\
\hline \multirow[t]{3}{*}{$5 \mathrm{~A}$} & Tris(2-methoxyphenyl) bismuth site A & 2 & -2.9 \\
\hline & & 3 & -4.2 \\
\hline & & 4 & -4.9 \\
\hline \multirow[t]{3}{*}{$7 \mathrm{~B}$} & Triphenylbismuth dichloride site B & 1 & -8.7 \\
\hline & & 2 & -8.7 \\
\hline & & 3 & -8.9 \\
\hline
\end{tabular}

A general error estimation to the data in Table VI and Table VII is given as follows.

Temperature accuracy:

$0.1 \mathrm{~K}$ (accuracy of temperature controller)

$0.5 \mathrm{~K}$ [experimental accuracy due to drift and offset (thermocouple position)]

NQR parameter:

At $310 \mathrm{~K}: \Delta \nu_{Q, k}=+/-10 \mathrm{kHz}$ (due to temperature stability during $1 \mathrm{~h}$ measurement)

$\Delta Q_{c c}:+/-0.4 \mathrm{MHz}$ (calculation variation within transition frequency limits)

$\Delta \eta:+/-0.001$ (calculation variation within transition frequency limits)

At $300 \mathrm{~K}$, at $(310 \mathrm{~K}): \Delta \nu_{Q, k}=+/-30 \mathrm{kHz}$ (extended sample-thermocouple distance) $\Delta Q_{c c}:+/-0.6 \mathrm{MHz}$ (calculation variation within transition frequency limits)

$\Delta \eta:+/-0.003$ (calculation variation within transition frequency limits)

At $77 \mathrm{~K}: \Delta \nu_{Q, k}=+/-5 \mathrm{kHz}$ (spectrometer accuracy)

$\Delta Q_{c c}:+/-0.2 \mathrm{MHz}$ (calculation variation within transition frequency limits)

$\Delta \eta:+/-0.001$ (calculation variation within transition frequency limits)

linear temperature coefficient $\mathrm{C}_{37}$

$+/-0.2 \mathrm{kHz} / \mathrm{K}$ (due to average deviation in $95 \%$ confidence bound)

Relaxation times:

Statistical fitting error:

$\Delta T_{1, Q}:<2 \%$ standard error (due to average deviation in $95 \%$ confidence bound)

$\Delta T_{2, Q}:<2 \%$ standard error (due to average deviation in $95 \%$ confidence bound)

Systematic error: (mainly due to temperature drift during the experiment and estimated from repeated $T_{2}$ and $T_{1}$ determination on a single transition, higher errors for low $T_{2}$ measurements due to low SNR)

At $300 \mathrm{~K} / 310 \mathrm{~K}$ : For $T_{2, Q}>200 \mu \mathrm{s}, \Delta T_{1, Q}:<10 \%, \Delta T_{2, Q}:<10 \%$

For $T_{2, Q}<200 \mu \mathrm{s}, \Delta T_{1, Q}:<20 \%, \Delta T_{2, Q}:<20 \%$

At $77 \mathrm{~K}$ : For $T_{2}>200 \mu \mathrm{s}, \Delta T_{1, Q}:<5 \%, \Delta T_{2, Q}:<5 \%$

For $T_{2}<200 \mu \mathrm{s}, \Delta T_{1, Q}:<10 \%, \Delta T_{2, Q}:<10 \%$

[1] R. B. Lauffer, Paramagnetic Metal Complexes as Water Proton Relaxation Agents for NMR Imaging: Theory and Design, Chem. Rev. 87, 901 (1987).

[2] G. J. Strijkers, W. J. M. Mulder, G. A. F. Van Tilborg, and K. Nicolay, MRI Contrast Agents: Current Status and
Future Perspectives, Anti-Cancer Agents Med. Chemistry 7, 291 (2007).

[3] A. R. Padhani, Dynamic Contrast-Enhanced MRI in Clinical Oncology: Current Status and Future Directions, J. Magn. Reson. Imaging 16, 407 (2002). 
[4] M. M. J. Modo and J. W. M. Bulte, Molecular and Cellular MR Imaging (CRC Press, Boca Raton, FL, 2007).

[5] Z.-Y. Chen et al., Advance of Molecular Imaging Technology and Targeted Imaging Agent in Imaging and Therapy, BioMed Res. Int. 2014, 819324 (2014).

[6] G.-L. Davies, I. Kramberger, and J. J. Davis, Environmentally Responsive MRI Contrast Agents, Chem. Commun. (Cambridge) 49, 9704 (2013).

[7] D. V. Hingorani, A. S. Bernstein, and M. D. Pagel, A Review of Responsive MRI Contrast Agents: 2005-2014, Contrast Media Mol. Imaging 10, 245 (2015).

[8] K. Ward, A. Aletras, and R. Balaban, A New Class of Contrast Agents for MRI Based on Proton Chemical Exchange Dependent Saturation Transfer (CEST), J. Magn. Reson. 143, 79 (2000).

[9] P. van Zijl and N. N. Yadav, Chemical Exchange Saturation Transfer (CEST): What Is in a Name and What Isn't?, Magn. Reson. Med. 65, 927 (2011).

[10] E. Vinogradov, A. D. Sherry, and R. E. Lenkinski, CEST: From Basic Principles to Applications, Challenges and Opportunities, J. Magn. Reson. 229, 155 (2013).

[11] H. V. T. Nguyen et al., Nitroxide-Based Macromolecular Contrast Agents with Unprecedented Transverse Relaxivity and Stability for Magnetic Resonance Imaging of Tumors, ACS Cent. Sci. 3, 800 (2017).

[12] G. Voigt and R. Kimmich, Quadrupolar Dip in Proton Relaxation Dispersion of Poly(vinyl Chloride), J. Magn. Reson. 24, 149 (1976).

[13] F. Winter and R. Kimmich, Spin Lattice Relaxation of Dipole Nuclei $(I=1 / 2)$ Coupled to Quadrupole Nuclei $(S=1)$, Mol. Phys. 45, 33 (1982).

[14] R. Kimmich, F. Winter, W. Nusser, and K. H. Spohn, Interactions and Fluctuations Deduced from Proton Field-Cycling Relaxation Spectroscopy of Polypeptides, DNA, Muscles, and Algae, J. Magn. Reson. 68, 263 (1986).

[15] P.-O. Westlund, The Quadrupole Enhanced 1H SpinLattice Relaxation of the Amide Proton in Slow Tumbling Proteins, Phys. Chem. Chem. Phys. 12, 3136 (2010).

[16] E. P. Sunde and B. Halle, Mechanism of 1H-14N CrossRelaxation in Immobilized Proteins, J. Magn. Reson. 203, 257 (2010).

[17] P. O. Westlund, Theoretical Reason for the Lack of Influence of 1 H-14N Cross-Relaxation on the Water Proton T 1 NMRD Profile in Slow Tumbling Proteins, Mol. Phys. 110, 2251 (2012).

[18] D. J. Lurie, S. Aime, S. Baroni, N. A. Booth, L. M. Broche, C.-H. Choi, G. R. Davies, S. Ismail, D. Ó. hÓgáin, and K. J. Pine, Fast Field-Cycling Magnetic Resonance Imaging, C.R. Phys. 11, 136 (2010).

[19] C. T. Harris, W. B. Handler, Y. Araya, F. MartínezSantiesteban, J. K. Alford, B. Dalrymple, F. Van Sas, B. A. Chronik, and T. J. Scholl, Development and Optimization of Hardware for Delta Relaxation Enhanced MRI, Magn. Reson. Med. 72, 1182 (2014).

[20] J. K. Alford, B. K. Rutt, T. J. Scholl, W. B. Handler, and B. A. Chronik, Delta Relaxation Enhanced MR: Improving Activation-Specificity of Molecular Probes through $R 1$ Dispersion Imaging, Magn. Reson. Med. 61, 796 (2009).
[21] U. C. Hoelscher, S. Lother, F. Fidler, M. Blaimer, and P. Jakob, Quantification and Localization of Contrast Agents Using Delta Relaxation Enhanced Magnetic Resonance at 1.5 T, Magnetic Resonance Materials in Physics, Biology, and Medicine 25, 223 (2012).

[22] Y. T. Araya, F. Martínez-Santiesteban, W. B. Handler, C. T. Harris, B. A. Chronik, and T. J. Scholl, Nuclear Magnetic Relaxation Dispersion of Murine Tissue for Development of $T_{1}\left(R_{1}\right)$ Dispersion Contrast Imaging, NMR Biomed. 30, e3789 (2017).

[23] D. Kruk, A. Kubica, W. Masierak, A. F. Privalov, M. Wojciechowski, and W. Medycki, Quadrupole Relaxation Enhancement-Application to Molecular Crystals, Solid State Nucl. Magn. Reson. 40, 114 (2011).

[24] G. G. Briand and N. Burford, Bismuth Compounds and Preparations with Biological or Medicinal Relevance, Chem. Rev. 99, 2601 (1999).

[25] R. J. McDonald, J. S. McDonald, D. F. Kallmes, M.E. Jentoft, M. A. Paolini, D. L. Murray, E. E. Williamson, and L. J. Eckel, Gadolinium Deposition in Human Brain Tissues after Contrast-Enhanced MR Imaging in Adult Patients without Intracranial Abnormalities, Radiology 285, 546 (2017).

[26] T. J. Fraum, D. R. Ludwig, M. R. Bashir, and K. J. Fowler, Gadolinium-Based Contrast Agents: A Comprehensive Risk Assessment, J. Magn. Reson. Imaging 46, 338 (2017).

[27] O. Rabin, J. M. Perez, J. Grimm, G. Wojtkiewicz, and R. Weissleder, An X-Ray Computed Tomography Imaging Agent Based on Long-Circulating Bismuth Sulphide Nanoparticles, Nat. Mater. 5, 118 (2006).

[28] A. Brown, Bismuth Nanoparticles as Medical X-Ray Contrast Agents: Synthesis, Characterization, and Applications, Diss. Thesis, Portland State University, 2013.

[29] P. C. Naha et al., Dextran Coated Bismuth-Iron Oxide Nanohybrid Contrast Agents for Computed Tomography and Magnetic Resonance Imaging, J. Mater. Chem. 2, 8239 (2014).

[30] T. P. Das and E. L. Hahn, Nuclear Quadrupole Resonance Spectroscopy, edited by F. Setz and D. Turn-bull, Solid State Physics (Academic Press, New York, 1958).

[31] G. B. Furman, V. M. Meerovich, and V. L. Sokolovsky, Nuclear Electric Quadrupole Interactions in Liquids Entrapped in Cavities, Hyperfine Interact. 237, 140 (2016).

[32] B. H. Suits, Nuclear Quadrupole Resonance Spectroscopy, Handbook of Applied Solid State Spectroscopy, edited by D. R. Vij (Springer, New York, 2006), Chap. 2.

[33] J. A. S. Smith, Nuclear Quadrupole Resonance Spectroscopy, J. Chem. Educ. 48, 39 (1971).

[34] R. Kubo, Stochastic Liouville Equations, J. Math. Phys. (N.Y.) 4, 174 (1963).

[35] P. O. Westlund and H. Wennerstrom, Spin-Lattice Relaxation of a Spin-1/2 Nucleus Coupled to a Quadrupolar Spin-1 Nucleus: The Quadrupolar Dip, J. Magn. Reson. 63, 280 (1985).

[36] A. G. Redfield, The Theory of Relaxation Processes, Adv. Magn. Opt. Reson. 1, 19 (1965).

[37] C. P. Slichter, Principles of Magnetic Resonance (Springer, Berlin, Heidelberg, 1990). 
[38] G. K. Semin, On Solving Secular Equations for HalfInteger Spins $(I=5 / 2,7 / 2$, and 9/2), Russ. J. Phys. Chem. 81, 38 (2007).

[39] H. Chihara and N. Nakamura, Nuclear Quadrupole Resonance Spectroscopy Data: Nuclei Zr...Bi, Diagrams, Structure formulas, Indexes, edited by K.-H. Hellwege and A. M. Hellwege, Landolt-Börnstein-Group III Condensed Matter Vol. 20C (Springer, 1989).

[40] M. A. McGuigan, Chronic Poisoning, in Goldman's Cecil Medicine (Elsevier, New York, 2012), pp. 88-95.

[41] S. Karelitz and A. D. Freedman, Hepatitis and Nephrosis Due to Soluble Bismuth, Pediatrics 8, 772 (1951).

[42] Y. Fujiwara, M. Mitani, S. Yasuike, J. Kurita, and T. Kaji, An Organobismuth Compound That Exhibits Selective Cytotoxicity to Vascular Endothelial Cells In Vitro, J. Heal. Sci. 51, 333 (2005).

[43] H. G. W. Robinson, H. G. Dehmelt, and W. Gordy, Pure Nuclear Quadrupole Spectrum of Bi209 in BismuthTriphenyl, Phys. Rev. 89, 1305 (1953).

[44] J. Weaver and F. N. H. Robinson, Nuclear Quadrupole Resonance of Bismuth and the Quadrupole Hamiltonian, Phys. Lett. 85A, 389 (1981).

[45] T. B. Brill and G. G. Long, Studies of Pentavalent Organoarsenic, -Antimony, and -Bismuth Halide Compounds by Nuclear Quadrupole Resonance Spectroscopy, Inorg. Chem. 9, 1980 (1970).

[46] R. F. van der Kelen and G. P. de Ketelaere, Organo Group $V B$ Chemistry. Part IV: On the NQR Spectra of Tertiary Aryl Group VB Compounds (35Cl, 75As,121Sb,209Bi), J. Mol. Struct. 23, 329 (1974).

[47] E. S. M. Lee, L. de Rochefort, G. Ferrante, and B. K. Rutt, Next Generation Delta Relaxation Enhanced MRI with \pm 0.36 T $\Delta$ B, Proc. Intl. Soc. Mag. Reson. Med. 20, 2877 (2012).

[48] M. Bödenler, M. Basini, M. F. Casula, E. Umut, C. Gösweiner, A. Petrovic, D. Kruk, and H. Scharfetter, $R$ 1 Dispersion Contrast at High Field with Fast FieldCycling MRI, J. Magn. Reson. 290, 68 (2018).

[49] G. Ferguson and E. W. Macaulay, The Stereochemistry of Some Organic Derivatives of Group VB Elements. Part III. The Crystal and Molecular Structure of Triphenylarsine Oxide Monohydrate, J. Chem. Soc. A, 1 (1969).

[50] O. Schuster, A. Schier, and H. Schmidbaur, The Quest for Complexes with a Coordinative Gold-Bismuth Bond, Organometallics 22, 4079 (2003).

[51] A. Weiss and S. Wigand, Correlation of NQR and Chemical Bond Parameter, Z. Naturforsch. 45A, 195 (1990).

[52] P. Lantto and J. Vaara, Calculations of Nuclear Quadrupole Coupling in Noble Gas-Noble Metal Fluorides: Interplay of Relativistic and Electron Correlation Effects, J. Chem. Phys. 125, 174315 (2006).

[53] M. Straka, P. Lantto, M. Räsänen, and J. Vaara, Theoretical Predictions of Nuclear Magnetic Resonance Parameters in a Novel Organo-Xenon Species: Chemical Shifts and Nuclear Quadrupole Couplings in $\mathrm{HXeCCH}$, J. Chem. Phys. 127, 234314 (2007).

[54] L. Belpassi, F. Tarantelli, A. Sgamellotti, H. M. Quiney, J. N.P. Van Stralen, and L. Visscher, Nuclear Electric Quadrupole Moment of Gold, J. Chem. Phys. 126, 064314 (2007).
[55] L. Cheng, S. Stopkowicz, and J. Gauss, Spin-Free DiracCoulomb Calculations Augmented with a Perturbative Treatment of Spin-Orbit Effects at the Hartree-Fock Level, J. Chem. Phys. 139, 214114 (2013).

[56] T. Q. Teodoro and R. L. A. Haiduke, Nuclear Electric Quadrupole Moment of Bismuth from the Molecular Method, Phys. Rev. A 88, 052504 (2013).

[57] A. Shee, L. Visscher, and T. Saue, Analytic One-Electron Properties at the 4-Component Relativistic Coupled Cluster Level with Inclusion of Spin-Orbit Coupling, J. Chem. Phys. 145, 184107 (2016).

[58] L. Cheng and J. Gauss, Perturbative Treatment of SpinOrbit Coupling within Spin-Free Exact Two-Component Theory, J. Chem. Phys. 141, 164107 (2014).

[59] J. Roukala, S. T. Orr, J. V. Hanna, J. Vaara, A. V. Ivanov, O. N. Antzutkin, and P. Lantto, Experimental and FirstPrinciples NMR Analysis of Pt(II) Complexes With O,O'Dialkyldithiophosphate Ligands, J. Phys. Chem. A 120, 8326 (2016).

[60] R. J. F. Berger, D. Rettenwander, S. Spirk, C. Wolf, M. Patzschke, M. Ertl, U. Monkowius, and N. W. Mitzel, Relativistic Effects in Triphenylbismuth and Their Influence on Molecular Structure and Spectroscopic Properties, Phys. Chem. Chem. Phys. 14, 15520 (2012).

[61] E. van Lenthe and E. J. Baerends, Density Functional Calculations of Nuclear Quadrupole Coupling Constants in the Zero-Order Regular Approximation for Relativistic Effects, J. Chem. Phys. 112, 8279 (2000).

[62] L. Visscher and E. van Lenthe, On the Distinction between Scalar, and Spin-Orbit Relativistic Effects, Chem. Phys. Lett. 306, 357 (1999).

[63] K. G. Dyall, An Exact Separation of the Spin-Free and Spin-Dependent Terms of the Dirac-Coulomb-Breit Hamiltonian, J. Chem. Phys. 100, 2118 (1994).

[64] O. V. Yazyev and L. Helm, Nuclear Spin Relaxation Parameters of MRI Contrast Agents-Insight from Quantum Mechanical Calculations, Eur. J. Inorg. Chem. 2008, 201 (2008).

[65] T. N. Rudakov, Nuclear Quadrupole Resonance, Instruments, in Encyclopedia of Spectroscopy and Spectrometry (Elsevier Ltd., New York, 2010), pp. 1966-1974.

[66] R. R. Gainov, A. V. Dooglav, I. A. Evlampiev, N. N. Mozgova, R. R. Khasanov, and Russian Federation, Determination of the Local Crystal-Chemical Features of Complex Chalcogenides by Copper, Antimony, and Arsenic NQR 4, 238 (2009).

[67] J. A. Lehmann-Horn, R. Yong, D. G. Miljak, and T. J. Bastow, 75As NQR Studies on FeAs2, Solid State Nucl. Magn. Reson. 71, 87 (2015).

[68] G. Koutroulakis, H. Yasuoka, P. H. Tobash, J. N. Mitchell, E. D. Bauer, and J.D. Thompson, Extended Nuclear Quadrupole Resonance Study of the Heavy-Fermion Superconductor PuCoGa5, Phys. Rev. B 94, 165115 (2016).

[69] A. P. Reyes, R. H. Heffner, P. C. Canfield, J. D. Thompson, and Z. Fisk, 209Bi NMR and NQR Investigation of the Small-Gap Semiconductor Ce3Bi4Pt3, Phys. Rev. B 49, 16321 (1994).

[70] N. Sinyavsky, E. Korotey, and M. Maćkowiak, Determination of the Electric Field Gradient Asymmetry from 
Berry's Phase in NQR of Powder Samples, J. Mol. Struct. 830, 131 (2007).

[71] H. Nakayama, T. Eguchi, and N. Nakamura, Phase Transitions in 4,4'-Dichlorobenzophenone as Studied by 3 SC1FT-NQR *, Z. Naturforsch. 49, 267 (1994).

[72] D. Possa, A. C. Gaudio, and J. C. C. Freitas, Numerical Simulation of NQR/NMR: Applications in Quantum Computing, J. Magn. Reson. 209, 250 (2011).

[73] C. Chen, F. Zhang, J. Barras, K. Althoefer, S. Bhunia, and S. Mandal, Authentication of Medicines Using Nuclear Quadrupole Resonance Spectroscopy, IEEE/ACM Trans. Comput. Biol. Bioinforma. 13, 417 (2016).

[74] H. Scharfetter, An Electronically Tuned Wideband Probehead for NQR Spectroscopy in the VHF Range, J. Magn. Reson. 271, 90 (2016).

[75] H. Scharfetter, M. Bödenler, and D. Narnhofer, A Cryostatic, Fast Scanning, Wideband NQR Spectrometer for the VHF Range, J. Magn. Reson. 286, 148 (2018).

[76] C. J. Pickard, P. J. Hasnip, M. J. Probert, K. Refson, and M. C. Payne, First Principles Methods using CASTEP, Z. Kristallogr. 220, 567 (2005).

[77] P. Jones, A. Blaschette, and D. Henschel, Redetermination of the Crystal Structure of Triphenylbismuth, (C6H5)3Bi, Z. Kristallogr. 210, 377 (1995).

[78] A. Tkatchenko and M. Scheffler, Accurate Molecular van der Waals Interactions from Ground-State Electron Density, and Free-Atom Reference Data, Phys. Rev. Lett. 102, 073005 (2009).

[79] D. Vanderbilt, Soft Self-Consistent Pseudopotentials in a Generalized Eigenvalue Formalism, Phys. Rev. B 41, 7892 (1990).

[80] E. J. Baerends et al., ADF2017, SCM, Theoretical Chemistry, Vrije Universiteit, Amsterdam, The Netherlands, https://www.scm.com/doc/ADF/Required_citations.html.

[81] C. F. Guerra, J. G. Snijders, G. te Velde, and E. J. Baerends, Towards an Order-N DFT Method, Theor. Chem. Acc. 99, 391 (1998).

[82] G. T. Velde, F. M. Bickelhaupt, E. J. Baerends, C. F. Guerra, S. J. A. van Gisbergen, J. G. Snijders, and T. Ziegler, Chemistry with ADF, J. Comput. Chem. 22, 931 (2001).

[83] J.P. Perdew, K. Burke, and M. Ernzerhof, Generalized Gradient Approximation Made Simple, Phys. Rev. Lett. 77, 3865 (1996).

[84] M. Ernzerhof and G. E. Scuseria, Assessment of the Perdew-Burke-Ernzerhof Exchange-Correlation Functional, J. Chem. Phys. 110, 5029 (1999).

[85] C. Adamo and V. Barone, Toward Reliable Density Functional Methods without Adjustable Parameters: The PBEO Model, J. Chem. Phys. 110, 6158 (1999).

[86] H. J. A. Jensen et al., DIRAC A Relativistic Ab Initio Electronic Structure Program, Release DIRAC16 (2016), http://www.diracprogram.org.

[87] E. Van Lenthe and E. J. Baerends, Optimized Slater-Type Basis Sets for the Elements 1-118, J. Comput. Chem. 24, 1142 (2003).
[88] T. H. Dunning, Gaussian Basis Sets for Use in Correlated Molecular Calculations. I. The Atoms Boron through Neon and Hydrogen, J. Chem. Phys. 90, 1007 (1989).

[89] T. Q. Teodoro, A. B. F. Da Silva, and R. L. A. Haiduke, Relativistic Prolapse-Free Gaussian Basis Set of

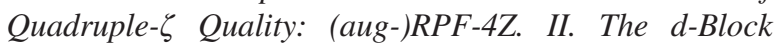
Elements, J. Chem. Theory Comput. 10, 4761 (2014).

[90] J. Bieroń and P. Pyykkö, Nuclear Quadrupole Moments of Bismuth, Phys. Rev. Lett. 87, 133003 (2001).

[91] M. H. Levitt, Spin Dynamics: Basics of Nuclear Magnetic Resonance 2nd ed. (Wiley, New York, 2007).

[92] A. Luqman, V. L. Blair, R. Brammananth, P. K. Crellin, R. L. Coppel, and P. C. Andrews, The Importance of Heterolepticity in Improving the Antibacterial Activity of Bismuth(III) Thiolates, Eur. J. Inorg. Chem. 2016, 2738 (2016).

[93] H. Suzuki and Y. Matano, Organobismuth Chemistry (Elsevier Science, New York, 2001).

[94] Martin Hébert, P. Petiot, E. Benoit, J. Dansereau, T. Ahmad, A. Le Roch, X. Ottenwaelder, and A. Gagnon, Synthesis of Highly Functionalized Triarylbismuthines by Functional Group Manipulation and Use in Palladiumand Copper-Catalyzed Arylation Reactions, J. Org. Chem. 81, 5401 (2016).

[95] A. Hassan, S. R. Breeze, S. Courtenay, C. Deslippe, and S. Wang, Organobismuth(III) and Organobismuth (V) Complexes Containing Pyridyl and Amino Functional Groups. Syntheses and Characterizations of $\mathrm{Bi}^{\mathrm{III}} \mathrm{Ar}_{3}\left(\mathrm{Ar}=\mathrm{p}-\mathrm{C}_{6} \mathrm{H}_{4}\left(\mathrm{NMe}_{2}\right), \mathrm{p}-\mathrm{C}_{6} \mathrm{H}_{4} \mathrm{CH}_{2}\left(\mathrm{NPr}{ }_{2}^{\mathrm{i}}\right)\right.$, $\mathrm{p}-$ $\left.\mathrm{C}_{6} \mathrm{H}_{4}\left[\mathrm{CH}_{2} \mathrm{~N}(2-\mathrm{Py})_{2}\right]\right), \quad \mathrm{Bi}^{\mathrm{V}} \mathrm{Ar}_{3} \mathrm{~L}_{2}, \quad\left[\mathrm{Bi}^{\mathrm{V}} \mathrm{Ar}_{3} \mathrm{Cl}\right]_{2} \mathrm{O}$, $\left[\mathrm{Bi}^{\mathrm{V}} \mathrm{Ar}_{4}\right]\left[\mathrm{PF}_{6}\right]$, and $\left[\mathrm{Bi}^{\mathrm{V}} \mathrm{Ar}_{4}\right]_{2}\left[\mathrm{Ag}_{2} \mathrm{Cl}_{4}\right] \quad(\mathrm{Ar}=\mathrm{p}-$ $\mathrm{C}_{6} \mathrm{H}_{4}\left(\mathrm{NMe}_{2}\right) \quad$ or $\quad \mathrm{p}-\mathrm{C}_{6} \mathrm{H}_{4}\left[\mathrm{CH}_{2} \mathrm{~N}(2-\mathrm{Py})_{2}\right], \quad \mathrm{L}=\mathrm{Cl}$, $\mathrm{CH}_{3} \mathrm{CO}_{2}{ }^{-}, \mathrm{CF}_{3} \mathrm{CO}_{2}{ }^{-}$), Organometallics 15, 5613 (1996).

[96] J. P. Parrish, T. V. Hughes, I. Hwang, and D. L. Boger, Establishing the Parabolic Relationship between Reactivity and Activity for Derivatives and Analogues of the Duocarmycin and CC-1065 Alkylation Subunits, J. Am. Chem. Soc. 126, 80 (2004).

[97] V. Sharutin, CCDC 929897: Experimental Crystal Structure Determination, CSD Commun., DOI 10.5517/ ccdc.csd.cc106mpj (2016).

[98] W. Levason, B. Sheikh, and F. P. McCullough, The Synthesis and Properties of Substituted Tertiary Bismuthine Ligands, J. Coord. Chem. 12, 53 (1982).

[99] T. Ogawa, T. Ikegami, T. Hikasa, N. Ono, and H. Suzuki, Enhanced Nucleophilicity of Tris-(2,6-dimethoxyphenyl) bismuthane as Studied by X-Ray Crystallography, 170 NM, J. Chem. Soc. Perkin Trans. 1, 3479 (1994).

[100] A. F. M. Mustafizu Rahman, T. Murafuji, M. Ishibashi, Y. Miyoshi, and Y. Sugihara, Effect of $\pi$-Accepting Substituent on the Reactivity and Spectroscopic Characteristics of Triarylbismuthanes and Triarylbismuth Dihalides, J. Organomet. Chem. 689, 3395 (2004).

[101] See Supplemental Material at http://link.aps.org/ supplemental/10.1103/PhysRevX.8.021076 for 3D structures of triphenylbismuth in crystalline and gas phase state. 